\title{
Generalized Trapezoidal Fuzzy Soft Set and Its Application in Medical Diagnosis
}

\author{
Haidong Zhang, ${ }^{1,2}$ Lan Shu, $^{2}$ and Shilong Liao ${ }^{1}$ \\ ${ }^{1}$ School of Mathematics and Computer Science, Northwest University for Nationalities, Lanzhou, Gansu 730030, China \\ ${ }^{2}$ School of Mathematical Sciences, University of Electronic Science and Technology of China, Chengdu, Sichuan 611731, China \\ Correspondence should be addressed to Haidong Zhang; lingdianstar@163.com
}

Received 11 December 2013; Revised 19 February 2014; Accepted 27 February 2014; Published 3 April 2014

Academic Editor: Fernando Simões

Copyright (c) 2014 Haidong Zhang et al. This is an open access article distributed under the Creative Commons Attribution License, which permits unrestricted use, distribution, and reproduction in any medium, provided the original work is properly cited.

Soft set theory is a newly emerging mathematical tool to deal with uncertain problems. In this paper, by introducing a generalization parameter, which itself is trapezoidal fuzzy, we define generalized trapezoidal fuzzy soft sets and then study some of their properties. Finally, applications of generalized trapezoidal fuzzy soft sets in a decision making problem and medical diagnosis problem are shown.

\section{Introduction}

Many complicated problems in economics, engineering, social sciences, medical sciences, and many other fields involve uncertain data. These problems, which one comes face to face with in life, cannot be solved using classical mathematic methods. There are several well-known theories to describe uncertainty, for instance, fuzzy set theory [1], rough set theory [2], and other mathematical tools. But all of these theories have their inherit difficulties as pointed out by Molodtsov [3]. To overcome these difficulties, in 1999 Molodtsov introduced the concept of soft sets, which can be seen as a new mathematical tool for dealing with uncertainties. This so-called soft set theory seems to be free from the difficulties affecting the existing methods. It has been found that fuzzy sets, rough sets, and soft sets are closely related concepts [4]. Soft set theory has potential applications in many different fields including the smoothness of functions, game theory, operational research, Perron integration, probability theory, and measurement theory $[3,5]$. Research works on soft sets are very active and progressing rapidly in these years. Maji et al. [6] defined several operations on soft sets and made a theoretical study on the theory of soft sets. Jun [7] introduced the notion of soft BCK/BCI-algebras. Jun and Park [8] discussed the applications of soft sets in ideal theory of BCK/BCI-algebras. Feng et al. [9] applied soft set theory to the study of semirings and initiated the notion of soft semirings. Furthermore, based on [6], Ali et al. [10] introduced some new operations on soft sets and improved the notion of complement of soft set. They proved that certain De Morgan's laws hold in soft set theory. Qin and Hong [11] introduced the notion of soft equality and established lattice structures and soft quotient algebras of soft sets. Park et al. [12] discussed some properties of equivalence soft set relations.

The study of hybrid models combining soft sets with other mathematical structures is emerging as an active research topic of soft set theory. Maji et al. [13] initiated the study on hybrid structures involving fuzzy sets and soft sets. They introduced the notion of fuzzy soft sets, which can be seen as a fuzzy generalization of soft sets. Furthermore, based on [13], Majumdar and Samanta [14] modified the definition of fuzzy soft sets and presented the notion of generalized fuzzy soft sets theory. Yang et al. [15] presented the concept of the interval-valued fuzzy soft sets by combining interval-valued fuzzy set $[16,17]$ and soft set models. By combining the multifuzzy set and soft set models, Yang et al. [18] presented the concept of the multifuzzy soft set and provided its application in decision making under an imprecise environment. Feng et al. [19] provided a framework to combine fuzzy sets, rough sets, and soft sets all together, which gave rise to several interesting new concepts such as rough soft sets, soft rough 
sets, and soft rough fuzzy sets. The combination of soft set and rough set models was also discussed by some researchers $[20,21]$.

The trapezoidal fuzzy number, as a vital concept of fuzzy set, is increasingly applied in many references [22, 23]. The membership function of a trapezoidal fuzzy number is piecewise linear and trapezoidal, which can express vagueness information caused by linguistic assessments through transforming them into numerical variables objectively. By combining the concepts of trapezoidal fuzzy sets and soft set models, Xiao et al. [24] presented the concept of the trapezoidal fuzzy soft sets which can deal with certain linguistic assessments. However, we can note that the attribute of the parameters in the soft set may be imprecise and vague such as the attribute "beautiful." In order to further capture the vagueness of the attribute with linguistic assessments information, it is natural for us to generalize the concept of the trapezoidal fuzzy soft sets as introduced by Xiao et al. [24]. In this paper, by introducing a generalization parameter, which itself is trapezoidal fuzzy, we define generalized trapezoidal fuzzy soft sets. In our generalization of trapezoidal fuzzy soft sets, a degree is attached to the parametrization of trapezoidal fuzzy sets while defining a trapezoidal fuzzy soft set. This definition is more realistic as it involves uncertainty in the selection of a trapezoidal fuzzy set corresponding to each value of the parameter. Some operations on the generalized trapezoidal fuzzy soft sets are also investigated. Then we present an example which shows that the decision making method of generalized trapezoidal fuzzy soft sets can be successfully applied to many problems that contain uncertainties. Finally, application of generalized trapezoidal fuzzy soft sets in a medical diagnosis problem is shown.

The rest of this paper is organized as follows. The following section briefly reviews some backgrounds on soft sets, trapezoidal fuzzy sets, and trapezoidal fuzzy soft sets. In Section 3, the concept of generalized trapezoidal fuzzy soft sets is presented. Some operations on the generalized trapezoidal fuzzy soft sets are then defined. Also some of their interesting properties are investigated. An application of generalized trapezoidal fuzzy soft sets in a medical diagnosis problem is shown in Section 4. Section 5 concludes the paper.

\section{Preliminaries}

The following definitions and preliminaries are required in the sequel of our work and hence presented in brief.

Definition 1 (see [3]). Let $U$ be an initial universe set and let $E$ be a universe set of parameters. A pair $(F, A)$ is called a soft set over $U$ if $A \subset E$ and $F: A \rightarrow P(U)$, where $P(U)$ is the set of all subsets of $U$.

Definition 2 (see [13]). Let $U$ be an initial universe set and let $E$ be a universe set of parameters. A pair $(F, A)$ is called a fuzzy soft set over $U$ if $A \subset E$ and $F: A \rightarrow F(U)$, where $F(U)$ is the set of all fuzzy subsets of $U$.

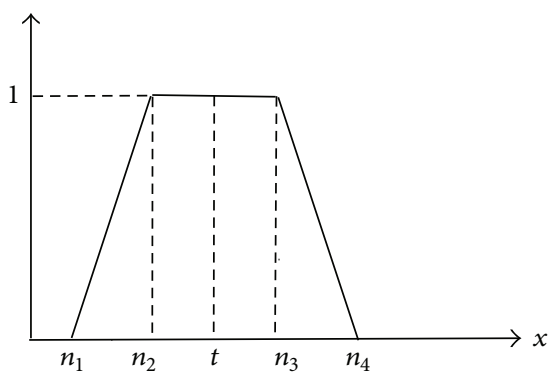

FIgURE 1: Trapezoidal fuzzy number $\tilde{n}$.

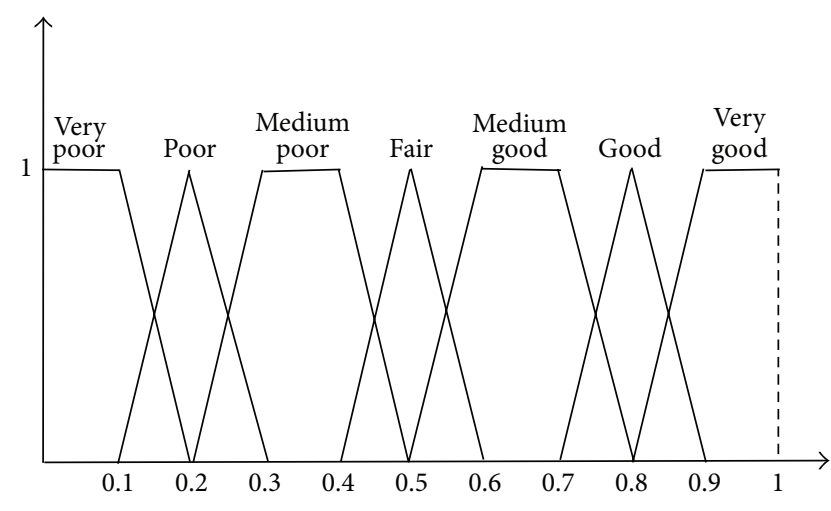

FIgURE 2: Linguistic variables for ratings.

Definition 3 (see [25]). A trapezoidal fuzzy number $\widetilde{n}$ can be defined as $\left(n_{1}, n_{2}, n_{3}, n_{4}\right)$ shown in Figure 1 which has the membership function $\mu_{\tilde{n}}(x)$ as follows:

$$
\mu_{\tilde{n}}(x)= \begin{cases}0, & x<n_{1}, \\ \frac{x-n_{1}}{n_{2}-n_{1}}, & n_{1} \leq x \leq n_{2} \\ 1, & n_{2} \leq x \leq n_{3} \\ \frac{x-n_{4}}{n_{3}-n_{4}}, & n_{3} \leq x \leq n_{4} \\ 0, & x>n_{4} .\end{cases}
$$

A set which is consisted by a trapezoidal fuzzy number or several trapezoidal fuzzy numbers is called trapezoidal fuzzy set, denoted by $\widetilde{I}$.

The membership function of a trapezoidal fuzzy number is piecewise linear and trapezoidal which can capture the vagueness of those linguistic assessments as in Figure 2. For example, the linguistic variable "medium poor" can be represented as $(0.2,0.3,0.4,0.5)$, the membership function of which is

$$
\mu_{\text {Medium poor }}(x)= \begin{cases}0, & x<0.2, \\ \frac{x-0.2}{0.3-0.2}, & 0.2 \leq x \leq 0.3, \\ 1, & 0.3 \leq x \leq 0.4, \\ \frac{x-0.5}{0.4-0.5}, & 0.4 \leq x \leq 0.5, \\ 0, & x>0.5 .\end{cases}
$$


TABLE 1: The ratings of five houses under various attributes.

\begin{tabular}{lccccc}
\hline$U$ & Cheap & Beautiful & Size & Location & In the green surroundings \\
\hline$u_{1}$ & Fair & Very good & Poor & Poor & Medium good \\
$u_{2}$ & Poor & Good & Good & Fair & Medium poor \\
$u_{3}$ & Good & Poor & Fair & Medium poor & Poor \\
$u_{4}$ & Medium poor & Medium good & Good & Fair & Good \\
$u_{5}$ & Medium Good & Medium poor & Poor & Good & Poor \\
\hline
\end{tabular}

Let $\widetilde{m}=\left(m_{1}, m_{2}, m_{3}, m_{4}\right)$ and $\widetilde{n}=\left(n_{1}, n_{2}, n_{3}, n_{4}\right)$ be two trapezoidal fuzzy numbers; then we have the following operations.

We can say $\widetilde{m} \leq \widetilde{n}[25]$ if and only if

$$
m_{1} \leq n_{1}, \quad m_{2} \leq n_{2}, \quad m_{3} \leq n_{3}, \quad m_{4} \leq n_{4} .
$$

The complement of $\widetilde{n}$ which is denoted by $\widetilde{n}^{c}$ can be defined as [25]

$$
\widetilde{n}^{c}=\left(1-n_{4}, 1-n_{3}, 1-n_{2}, 1-n_{1}\right) .
$$

The union of $\widetilde{m}$ and $\widetilde{n}$ which is denoted by $\widetilde{m} \cup \widetilde{n}$ can be defined as [25]

$$
\widetilde{z}=\widetilde{m} \cup \widetilde{n}=\left(m_{1} \vee n_{1}, m_{2} \vee n_{2}, m_{3} \vee n_{3}, m_{4} \vee n_{4}\right) .
$$

The intersection of $\widetilde{m}$ and $\widetilde{n}$ which is denoted by $\widetilde{m} \cap \widetilde{n}$ can be defined as [25]

$$
\widetilde{z}=\widetilde{m} \cap \widetilde{n}=\left(m_{1} \wedge n_{1}, m_{2} \wedge n_{2}, m_{3} \wedge n_{3}, m_{4} \wedge n_{4}\right) .
$$

The multiplication of $\widetilde{m}$ and $\widetilde{n}$ which is denoted by $\widetilde{m} \otimes \widetilde{n}$ can be defined as [26]

$$
\widetilde{z}=\widetilde{m} \otimes \widetilde{n}=\left(m_{1} \times n_{1}, m_{2} \times n_{2}, m_{3} \times n_{3}, m_{4} \times n_{4}\right) .
$$

Next the defuzzification method of a trapezoidal fuzzy number will be introduced (see [27]). Take a trapezoidal fuzzy number parameterized by a quadruplet $\left(n_{1}, n_{2}, n_{3}, n_{4}\right)$ as shown in Figure 1.

Then the defuzzification value $t$ of the trapezoidal fuzzy number is calculated from the figure as follows:

$$
\begin{aligned}
\left(t-n_{2}\right)+\frac{1}{2}\left(n_{2}-n_{1}\right) & =\left(n_{3}-t\right)+\frac{1}{2}\left(n_{4}-n_{3}\right) \Longrightarrow t \\
& =\frac{n_{1}+n_{2}+n_{3}+n_{4}}{4} .
\end{aligned}
$$

By using the concepts of trapezoidal fuzzy sets and soft sets, Xiao et al. [24] presented the concept of trapezoidal fuzzy soft sets.

Definition 4 (see [24]). Let $T F(U)$ be the set of all trapezoidal fuzzy subsets of $U$. A pair $(\widetilde{F}, A)$ is called a trapezoidal fuzzy soft set over $U$, where $\widetilde{F}$ is a mapping given by $\widetilde{F}: A \rightarrow$ $T F(U)$.

Example 5 (see [24]). Let $U$ be a set of five houses under consideration of a decision maker to purchase, which is denoted by $U=\left\{u_{1}, u_{2}, u_{3}, u_{4}, u_{5}\right\}$. Let $E$ be a parameter set, where $E=\left\{e_{1}, e_{2}, e_{3}, e_{4}, e_{5}\right\}=\{$ cheap; beautiful; size; location; in the green surroundings\}. Someone describes the optional five houses under various attributes with linguistic variables intuitively as in Table 1.

Then, we can have a corresponding trapezoidal fuzzy soft set $(\widetilde{F}, A)$ over the universe $U$ through the rule of conversion between linguistic variables and numerical variables showed in Figure 2.

Consider

$$
\begin{aligned}
\widetilde{F}\left(e_{1}\right)= & \left\{\frac{u_{1}}{(0.4,0.5,0.5,0.6)}, \frac{u_{2}}{(0.1,0.2,0.2,0.3)},\right. \\
& \frac{u_{3}}{(0.7,0.8,0.8,0.9)}, \frac{u_{4}}{(0.2,0.3,0.4,0.5)}, \\
\widetilde{F}\left(e_{2}\right)= & \left\{\frac{u_{5}}{(0.5,0.6,0.7,0.8)}\right\}, \\
& \frac{u_{1}}{(0.8,0.9,1.0,1.0)}, \frac{u_{2}}{(0.1,0.2,0.2,0.3)}, \frac{u_{3}}{(0.5,0.6,0.7,0.8)}, \\
& \left.\frac{u_{5}}{(0.2,0.3,0.4,0.5)}\right\},
\end{aligned}
$$

$$
\begin{aligned}
& \widetilde{F}\left(e_{3}\right)=\left\{\frac{u_{1}}{(0.1,0.2,0.2,0.3)}, \frac{u_{2}}{(0.7,0.8,0.8,0.9)},\right. \\
& \frac{u_{3}}{(0.4,0.5,0.5,0.6)}, \frac{u_{4}}{(0.7,0.8,0.8,0.9)} \text {, }
\end{aligned}
$$$$
\left.\frac{u_{5}}{(0.1,0.2,0.2,0.3)}\right\} \text {, }
$$$$
\widetilde{F}\left(e_{4}\right)=\left\{\frac{u_{1}}{(0.1,0.2,0.2,0.3)}, \frac{u_{2}}{(0.4,0.5,0.5,0.6)},\right.
$$$$
\frac{u_{3}}{(0.2,0.3,0.4,0.5)}, \frac{u_{4}}{(0.4,0.5,0.5,0.6)} \text {, }
$$$$
\left.\frac{u_{5}}{(0.7,0.8,0.8,0.9)}\right\} \text {, }
$$

$$
\begin{aligned}
\widetilde{F}\left(e_{5}\right)=\left\{\frac{u_{1}}{(0.5,0.6,0.7,0.8)}, \frac{u_{2}}{(0.2,0.3,0.4,0.5)},\right. \\
\frac{u_{3}}{(0.1,0.2,0.2,0.3)}, \frac{u_{4}}{(0.7,0.8,0.8,0.9)}, \\
\left.\frac{u_{5}}{(0.1,0.2,0.2,0.3)}\right\} .
\end{aligned}
$$


Remark 6. In Example 5, we can note that the attribute of the parameters in the trapezoidal fuzzy soft sets is imprecise and vague such as the attribute "beautiful." However, Xiao et al. do not point out how to depict the vague attribute of the parameters at all. So the model has its disadvantages which do not solve some problems that contain uncertainties when the attribute of the parameters in the trapezoidal fuzzy soft sets is imprecise and vague. In order to overcome the difficulty, we will introduce a generalization parameter, which itself is trapezoidal fuzzy, and present the concept of generalized trapezoidal fuzzy soft sets in the following section.

\section{Generalized Trapezoidal Fuzzy Soft Sets}

3.1. Concept of Generalized Trapezoidal Fuzzy Soft Sets. In this subsection, we generalize the concept of trapezoidal fuzzy soft sets as introduced by Xiao et al. [24]. In our generalization of trapezoidal fuzzy soft sets, a degree is attached to the parametrization of trapezoidal fuzzy sets while defining a trapezoidal fuzzy soft set.

Definition 7. Let $U$ be an initial universe and let $E$ be a set of parameters. The pair $(U, E)$ is called a soft universe. Suppose that $\widetilde{F}: E \rightarrow T F(U)$ and $\widetilde{f}$ is a trapezoidal fuzzy subset of $E$; that is, $\widetilde{f}: E \rightarrow \widetilde{I}$. We say that $\widetilde{F}_{\tilde{f}}$ is a generalized trapezoidal fuzzy soft set (GTFSS, in short) over the soft universe $(U, E)$ if and only if $\widetilde{F}_{\tilde{f}}$ is a mapping given by

$$
\widetilde{F}_{\tilde{f}}: E \longrightarrow T F(U) \times \widetilde{I}
$$

where $\widetilde{F}_{\tilde{f}}(e)=(\widetilde{F}(e), \widetilde{f}(e))$, such that for all $e \in E, \widetilde{F}(e) \in$ $T F(U)$, and $\widetilde{f}(e) \in \widetilde{I}$.

Here for each parameter $e_{i}, \widetilde{F}_{\tilde{f}}\left(e_{i}\right)=\left(\widetilde{F}\left(e_{i}\right), \widetilde{f}\left(e_{i}\right)\right)$ indicates not only the trapezoidal fuzzy membership degree of belongingness of the elements of $U$ in $F\left(e_{i}\right)$ but also the trapezoidal fuzzy membership degree of possibility of such belongingness of the parameters of $E$ which is represented by $\widetilde{f}\left(e_{i}\right)$.

We can note that a generalized trapezoidal fuzzy soft set $\widetilde{F}_{\tilde{f}}$ is actually a soft set because it is still a mapping from parameters to $T F(U) \times \tilde{I}$, and it can be written as

$$
\widetilde{F}_{\tilde{f}}(e)=(\widetilde{F}(e), \widetilde{f}(e)),
$$

where

$$
\begin{gathered}
\widetilde{F}(e)=\left\{\frac{u}{\left(\mu_{\widetilde{F}(e)}^{1}(u), \mu_{\widetilde{F}(e)}^{2}(u), \mu_{\widetilde{F}(e)}^{3}(u), \mu_{\widetilde{F}(e)}^{4}(u)\right)}: u \in U\right\}, \\
\widetilde{f}(e)=\left(\mu_{\widetilde{f}(e)}^{1}, \mu_{\widetilde{f}(e)}^{2}, \mu_{\widetilde{f}(e)}^{3}, \mu_{\widetilde{f}(e)}^{4}\right) .
\end{gathered}
$$

Sometimes we write $\widetilde{F}_{\tilde{f}}$ as $\left(\widetilde{F}_{\tilde{f}}, E\right)$. If $A \subset E$, we can also have a GTFSS $\left(\widetilde{F}_{\tilde{f}}, A\right)$.
TABLE 2: The ratings of five houses under various attributes for $\mathrm{Mr}$ $\mathrm{X}$.

\begin{tabular}{lccc}
\hline$U$ & Location & Cheap & Size \\
\hline$u_{1}$ & Medium good & Medium poor & Poor \\
$u_{2}$ & Poor & Good & Good \\
$u_{3}$ & Very poor & Medium poor & Fair \\
$u_{4}$ & Fair & Fair & Medium good \\
$u_{5}$ & Good & Poor & Poor \\
$\widetilde{f}$ & Fair & Medium good & Medium poor \\
\hline
\end{tabular}

Example 8. Suppose that a married couple, Mr. X and Mrs. $\mathrm{X}$, come to the real estate agent to buy a house. Assume that a real estate agent has a set of different types of houses $U=\left\{u_{1}, u_{2}, u_{3}, u_{4}, u_{5}\right\}$ which may be characterized by a set of parameters $E=\left\{e_{1}, e_{2}, e_{3}\right\}$. For $j=1,2,3$ the parameters $x_{j}$ stand for "location," "cheap," and "size," respectively. Considering his own needs, Mr. X describes the optional five houses under various attributes with linguistic variables intuitively as in Table 2 .

Then, we can have a corresponding generalized trapezoidal fuzzy soft set $\widetilde{F}_{\tilde{f}}$ over the universe $(U, E)$ through the rule of conversion between linguistic variables and numerical variables showed in Figure 2.

Consider

$$
\begin{gathered}
\widetilde{F}_{\tilde{f}}\left(e_{1}\right)=\left(\left\{\frac{u_{1}}{(0.5,0.6,0.7,0.8)}, \frac{u_{2}}{(0.1,0.2,0.2,0.3)},\right.\right. \\
\frac{u_{3}}{(0.0,0.1,0.1,0.2)}, \frac{u_{4}}{(0.4,0.5,0.5,0.6)}, \\
\widetilde{F}_{\tilde{f}}\left(e_{2}\right)=\left(\left\{\frac{u_{5}}{(0.7,0.8,0.8,0.9)}\right\},(0.4,0.5,0.5,0.6)\right), \\
\widetilde{F}_{\tilde{f}}\left(e_{3}\right)=\left(\left\{\frac{u_{1}}{(0.2,0.3,0.4,0.5)}, \frac{u_{2}}{(0.2,0.3,0.4,0.5)}, \frac{u_{3}}{(0.4,0.5,0.5,0.0 .6)},\right.\right. \\
\frac{\left.\frac{u_{5}}{(0.1,0.2,0.2,0.3)}\right\},(0.5,0.6,0.7,0.8)}{(0.1,0.2,0.2,0.3)}, \frac{u_{1}}{(0.7,0.8,0.8,0.9)}, \\
\frac{u_{3}}{(0.4,0.5,0.5,0.6)}, \frac{u_{4}}{(0.5,0.6,0.7,0.8)}, \\
\left.\left.\frac{u_{5}}{(0.1,0.2,0.2,0.3)}\right\},(0.2,0.3,0.4,0.5)\right) .
\end{gathered}
$$

Remark 9. In Example 8, we consider the vagueness of the attribute of the parameters. For example, the attribute "cheap" is imprecise and is characterized by a trapezoidal fuzzy number $(0.5,0.6,0.7,0.8)$. But as we have pointed out before, in Example 5 the vagueness of the attribute of the parameters is not considered. So the difference between the trapezoidal fuzzy soft set and the generalized trapezoidal fuzzy soft set is whether the vagueness of the attribute of the parameters 
has been depicted or not. Compared with the trapezoidal fuzzy soft set, the generalized trapezoidal fuzzy soft set can further capture the vagueness of the attribute with linguistic assessments information.

Definition 10. Let $\widetilde{F}_{\tilde{f}}$ and $\widetilde{G}_{\tilde{g}}$ be two GTFSSs over $(U, E)$. Now $\widetilde{F}_{\tilde{f}}$ is said to be a generalized trapezoidal fuzzy soft subset of $\widetilde{G}_{\widetilde{g}}$ if and only if

(1) for all $e \in E$, trapezoidal fuzzy numbers $\tilde{f}(e) \leq \widetilde{g}(e)$; that is, $\mu_{\tilde{f}(e)}^{1} \leq \mu_{\tilde{g}(e)}^{1}, \mu_{\tilde{f}(e)}^{2} \leq \mu_{\tilde{g}(e)}^{2}, \mu_{\tilde{f}(e)}^{3} \leq \mu_{\tilde{g}(e)}^{3}$, and $\mu_{\tilde{f}(e)}^{4} \leq \mu_{\widetilde{g}(e)}^{4} ;$

(2) for all $e \in E, \widetilde{F}(e)$ is a trapezoidal fuzzy subset of $\widetilde{G}(e)$; that is, $\mu_{\widetilde{F}(e)}^{1}(u) \leq \mu_{\widetilde{G}(e)}^{1}(u), \mu_{\widetilde{F}(e)}^{2}(u) \leq \mu_{\widetilde{G}(e)}^{2}(u)$, $\mu_{\widetilde{F}(e)}^{3}(u) \leq \mu_{\widetilde{G}(e)}^{3}(u)$, and $\mu_{\widetilde{F}(e)}^{4}(u) \leq \mu_{\widetilde{G}(e)}^{4}(u)$ in $\widetilde{F}(e)$ and $\widetilde{G}(e)$ over $U$.

In this case, we write $\widetilde{F}_{\tilde{f}} \sqsubseteq \widetilde{G}_{\tilde{g}}$.

Example 11. Consider the GTFSS $\widetilde{F}_{\tilde{f}}$ over $(U, E)$ given in Example 8. Let $\widetilde{G}_{\tilde{g}}$ be another GTFSS over $(U, E)$ defined as follows:

$$
\begin{gathered}
\widetilde{G}_{\tilde{g}}\left(e_{1}\right)=\left(\left\{\frac{u_{1}}{(0.2,0.3,0.4,0.5)}, \frac{u_{2}}{(0.0,0.1,0.1,0.2)},\right.\right. \\
\frac{u_{3}}{(0.0,0.1,0.1,0.2)}, \frac{u_{4}}{(0.2,0.3,0.4,0.5)}, \\
\widetilde{G}_{\tilde{g}}\left(e_{2}\right)=\left(\left\{\frac{u_{5}}{(0.5,0.6,0.7,0.8)}\right\},(0.2,0.3,0.4,0.5)\right), \\
\widetilde{G}_{\tilde{g}}\left(e_{3}\right)=\left(\left\{\frac{u_{1}}{(0.1,0.2,0.2,0.3)}, \frac{u_{2}}{(0.2,0.3,0.4,0.5)}, \frac{u_{3}}{(0.2,0.3,0.4,0.5)},\right.\right. \\
\left.\left.\frac{u_{5}}{(0.1,0.2,0.2,0.3)}\right\},(0.0,0.1,0.1,0.2)\right), \\
\frac{u_{1}}{(0.2,0.3,0.4,0.5)}, \frac{u_{2}}{(0.4,0.5,0.5,0.6)}, \\
\left.\left.\frac{u_{3}}{(0.1,0.2,0.2,0.3)}\right\},(0.2,0.3,0.4,0.5)\right) .
\end{gathered}
$$

Clearly, we have $\widetilde{G}_{\tilde{g}} \sqsubseteq \widetilde{F}_{\tilde{f}}$.

Definition 12. Let $\widetilde{F}_{\tilde{f}}$ and $\widetilde{G}_{\tilde{g}}$ be two GTFSSs over $(U, E)$. Now $\widetilde{F}_{\tilde{f}}$ and $\widetilde{G}_{\tilde{g}}$ are said to be a generalized trapezoidal fuzzy soft equal if and only if

(1) $\widetilde{F}_{\widetilde{f}}$ is a generalized trapezoidal fuzzy soft subset of $\widetilde{G}_{\widetilde{g}}$;

(2) $\widetilde{G}_{\widetilde{g}}$ is a generalized trapezoidal fuzzy soft subset of $\widetilde{F}_{\widetilde{f}}$, which can be denoted by $\widetilde{F}_{\tilde{f}}=\widetilde{G}_{\tilde{g}}$.

\subsection{Operations on Generalized Trapezoidal Fuzzy Soft Sets}

Definition 13. Let $\widetilde{F}_{\widetilde{f}}$ be a GTFSS over $(U, E)$. Then the complement of $\widetilde{F}_{\tilde{f}}$, denoted by $\widetilde{F}_{\tilde{f}}^{c}$, is defined by $\widetilde{F}_{\tilde{f}}^{c}=\widetilde{G}_{\tilde{g}}$, where $\widetilde{G}(e)=\widetilde{F}^{c}(e)=\left\{u /\left(1-\mu_{\widetilde{F}(e)}^{4}(u), 1-\mu_{\widetilde{F}(e)}^{3}(u), 1-\right.\right.$ $\left.\left.\mu_{\widetilde{F}(e)}^{2}(u), 1-\mu_{\widetilde{F}(e)}^{1}(u)\right): u \in U\right\}$ and $\widetilde{g}(e)=\widetilde{f}^{c}(e)=(1-$ $\left.\mu_{\widetilde{f}(e)}^{4}, 1-\mu_{\widetilde{f}(e)}^{3}, 1-\mu_{\widetilde{f}(e)}^{2}, 1-\mu_{\widetilde{f}(e)}^{1}\right)$.

From the above definition, we can see that $\left(\widetilde{F}_{\widetilde{f}}^{c}\right)^{c}=\widetilde{F}_{\tilde{f}}$.

Example 14. Consider the GTFSS $\widetilde{G}_{\widetilde{g}}$ over $(U, E)$ defined in Example 11. Thus, by Definition 13, we have

$$
\begin{aligned}
& \widetilde{G}_{\widetilde{g}}^{c}\left(e_{1}\right)=\left(\left\{\frac{u_{1}}{(0.5,0.6,0.7,0.8)}, \frac{u_{2}}{(0.8,0.9,0.9,1.0)},\right.\right. \\
& \frac{u_{3}}{(0.8,0.9,0.9,1.0)}, \frac{u_{4}}{(0.5,0.6,0.7,0.8)}, \\
& \left.\left.\frac{u_{5}}{(0.2,0.3,0.4,0.5)}\right\},(0.5,0.6,0.7,0.8)\right) \text {, } \\
& \widetilde{G}_{\widetilde{g}}^{c}\left(e_{2}\right)=\left(\left\{\frac{u_{1}}{(0.7,0.8,0.8,0.9)}, \frac{u_{2}}{(0.2,0.3,0.4,0.5)},\right.\right. \\
& \frac{u_{3}}{(0.5,0.6,0.7,0.8)}, \frac{u_{4}}{(0.5,0.6,0.7,0.8)}, \\
& \left.\left.\frac{u_{5}}{(0.7,0.8,0.8,0.9)}\right\},(0.8,0.9,0.9,1.0)\right) \text {, } \\
& \widetilde{G}_{\widetilde{g}}^{c}\left(e_{3}\right)=\left(\left\{\frac{u_{1}}{(0.8,0.9,0.9,1.0)}, \frac{u_{2}}{(0.1,0.2,0.2,0.3)},\right.\right. \\
& \frac{u_{3}}{(0.5,0.6,0.7,0.8)}, \frac{u_{4}}{(0.4,0.5,0.5,0.6)}, \\
& \left.\left.\frac{u_{5}}{(0.7,0.8,0.8,0.9)}\right\},(0.5,0.6,0.7,0.8)\right) \text {. }
\end{aligned}
$$

Definition 15. The union operation on the two GTFSSs $\widetilde{F}_{\tilde{f}}$ and $\widetilde{G}_{\tilde{g}}$, denoted by $\widetilde{F}_{\tilde{f}} \widetilde{\sqcup} \widetilde{G}_{\tilde{g}}$, is defined by a mapping given by $\widetilde{H}_{\tilde{h}}$ : $E \rightarrow T F(U) \times \widetilde{I}$, such that for all $u \in U \widetilde{H}_{\widetilde{h}}(e)=(\widetilde{H}(e), \widetilde{h}(e))$, where $\widetilde{H}(e)=\widetilde{F}(e) \sqcup \widetilde{G}(e)=\left\{u / \mu_{\widetilde{F}(e)}(u) \cup \mu_{\widetilde{G}(e)}(u): u \in\right.$ $U\}=\left\{u /\left(\mu_{\widetilde{F}(e)}^{1}(u) \vee \mu_{\widetilde{G}(e)}^{1}(u), \mu_{\widetilde{F}(e)}^{2}(u) \vee \mu_{\widetilde{G}(e)}^{2}(u), \mu_{\widetilde{F}(e)}^{3}(u) \vee\right.\right.$ $\left.\left.\mu_{\widetilde{G}(e)}^{3}(u), \mu_{\widetilde{F}(e)}^{4}(u) \vee \mu_{\widetilde{G}(e)}^{4}(u)\right): u \in U\right\}$ and $\widetilde{h}(e)=\widetilde{f}(e) \cup \widetilde{g}(e)=$ $\left(\mu_{\widetilde{f}(e)}^{1} \vee \mu_{\tilde{g}(e)}^{1}, \mu_{\widetilde{f}(e)}^{2} \vee \mu_{\tilde{g}(e)}^{2}, \mu_{\tilde{f}(e)}^{3} \vee \mu_{\tilde{g}(e)}^{3}, \mu_{\tilde{f}(e)}^{4} \vee \mu_{\tilde{g}(e)}^{4}\right)$.

Definition 16. The intersection operation on the two GTFSSs $\widetilde{F}_{\tilde{f}}$ and $\widetilde{G}_{\tilde{g}}$, denoted by $\widetilde{F}_{\tilde{f}} \widetilde{\Pi}_{G_{\tilde{g}}}$, is defined by a mapping given by $\widetilde{H}_{\widetilde{h}}: E \rightarrow T F(U) \times \widetilde{I}$, such that for all $u \in U \widetilde{H}_{\widetilde{h}}(e)=$ $(\widetilde{H}(e), \widetilde{h}(e))$, where $\widetilde{H}(e)=\widetilde{F}(e) \sqcap \widetilde{G}(e)=\left\{u / \mu_{\widetilde{F}(e)}(u) \cap\right.$ $\left.\mu_{\widetilde{G}(e)}(u): u \in U\right\}=\left\{u /\left(\mu_{\widetilde{F}(e)}^{1}(u) \wedge \mu_{\widetilde{G}(e)}^{1}(u), \mu_{\widetilde{F}(e)}^{2}(u) \wedge\right.\right.$ $\left.\left.\mu_{\widetilde{G}(e)}^{2}(u), \mu_{\widetilde{F}(e)}^{3}(u) \wedge \mu_{\widetilde{G}(e)}^{3}(u), \mu_{\widetilde{F}(e)}^{4}(u) \wedge \mu_{\widetilde{G}(e)}^{4}(u)\right): u \in U\right\}$ 
and $\widetilde{h}(e)=\tilde{f}(e) \cap \tilde{g}(e)=\left(\mu_{\tilde{f}(e)}^{1} \wedge \mu_{\tilde{g}(e)}^{1}, \mu_{\tilde{f}(e)}^{2} \wedge \mu_{\tilde{g}(e)}^{2}, \mu_{\tilde{f}(e)}^{3} \wedge\right.$ $\left.\mu_{\tilde{g}(e)}^{3}, \mu_{\tilde{f}(e)}^{4} \wedge \mu_{\tilde{g}(e)}^{4}\right)$.

Example 17. Let us consider the GTFSS $\widetilde{F}_{\widetilde{f}}$ in Example 8. Let $\widetilde{G}_{\tilde{g}}$ be another GTFSS over $(U, E)$ defined as follows:

$$
\begin{gathered}
\widetilde{G}_{\tilde{g}}\left(e_{1}\right)=\left(\left\{\frac{u_{1}}{(0.4,0.5,0.5,0.6)}, \frac{u_{2}}{(0.0,0.1,0.1,0.2)},\right.\right. \\
\frac{u_{3}}{(0.7,0.8,0.8,0.9)}, \frac{u_{4}}{(0.5,0.6,0.7,0.8)}, \\
\widetilde{G}_{\tilde{g}}\left(e_{2}\right)=\left(\left\{\frac{u_{5}}{(0.8,0.9,1.0,1.0)}\right\},(0.5,0.6,0.7,0.8)\right), \\
\quad \frac{u_{1}}{(0.4,0.5,0.5,0.6)}, \frac{u_{2}}{(0.2,0.3,0.4,0.5)}, \\
\left.\widetilde{G}_{\tilde{g}}\left(e_{3}\right)=\left(\frac{u_{3}}{(0.0,0.6,0.7,0.8)}, \frac{u_{5}}{(0.2,0.1,0.0 .4)}\right\},(0.2,0.3,0.4,0.5)\right), \\
\left\{\frac{u_{1}}{(0.5,0.6,0.7,0.8)}, \frac{u_{2}}{(0.5,0.6,0.7,0.8)},\right. \\
\frac{u_{3}}{(0.7,0.8,0.8,0.9)}, \frac{u_{4}}{(0.2,0.3,0.4,0.5)}, \\
\left.\left.\frac{u_{5}}{(0.4,0.5,0.5,0.6)}\right\},(0.7,0.8,0.8,0.9)\right) .
\end{gathered}
$$

Then by Definition 15, we have

$$
\begin{aligned}
& \left(\widetilde{F}_{\tilde{f}} \widetilde{\sqcup} \widetilde{G}_{\tilde{g}}\right)\left(e_{1}\right)=\left(\left\{\frac{u_{1}}{(0.5,0.6,0.7,0.8)}, \frac{u_{2}}{(0.1,0.2,0.2,0.3)},\right.\right. \\
& \frac{u_{3}}{(0.7,0.8,0.8,0.9)}, \frac{u_{4}}{(0.5,0.6,0.7,0.8)}, \\
& \left.\left.\frac{u_{5}}{(0.8,0.9,1.0,1.0)}\right\},(0.5,0.6,0.7,0.8)\right) \text {, } \\
& \left(\widetilde{F}_{\tilde{f}} \widetilde{\sqcup} \widetilde{G}_{\tilde{g}}\right)\left(e_{2}\right)=\left(\left\{\frac{u_{1}}{(0.4,0.5,0.5,0.6)}, \frac{u_{2}}{(0.7,0.8,0.8,0.9)},\right.\right. \\
& \frac{u_{3}}{(0.5,0.6,0.7,0.8)}, \frac{u_{4}}{(0.4,0.5,0.5,0.6)}, \\
& \left.\left.\frac{u_{5}}{(0.1,0.2,0.2,0.3)}\right\},(0.5,0.6,0.7,0.8)\right) \text {, } \\
& \left(\widetilde{F}_{\tilde{f}} \widetilde{\square} \widetilde{G}_{\tilde{g}}\right)\left(e_{3}\right)=\left(\left\{\frac{u_{1}}{(0.5,0.6,0.7,0.8)}, \frac{u_{2}}{(0.7,0.8,0.8,0.9)},\right.\right. \\
& \frac{u_{3}}{(0.7,0.8,0.8,0.9)}, \frac{u_{4}}{(0.5,0.6,0.7,0.8)}, \\
& \left.\left.\frac{u_{5}}{(0.4,0.5,0.5,0.6)}\right\},(0.7,0.8,0.8,0.9)\right)
\end{aligned}
$$

By Definition 16, then

$$
\begin{aligned}
& \left(\widetilde{F}_{\tilde{f}} \widetilde{\sqcap} \widetilde{G}_{\tilde{g}}\right)\left(e_{1}\right)=\left(\left\{\frac{u_{1}}{(0.4,0.5,0.5,0.6)}, \frac{u_{2}}{(0.0,0.1,0.1,0.2)},\right.\right. \\
& \frac{u_{3}}{(0.0,0.1,0.1,0.2)}, \frac{u_{4}}{(0.4,0.5,0.5,0.6)}, \\
& \left.\left.\frac{u_{5}}{(0.7,0.8,0.8,0.9)}\right\},(0.4,0.5,0.5,0.6)\right) \text {, } \\
& \left(\widetilde{F}_{\tilde{f}} \widetilde{\Pi} \widetilde{G}_{\widetilde{g}}\right)\left(e_{2}\right)=\left(\left\{\frac{u_{1}}{(0.2,0.3,0.4,0.5)}, \frac{u_{2}}{(0.2,0.3,0.4,0.5)},\right.\right. \\
& \frac{u_{3}}{(0.2,0.3,0.4,0.5)}, \frac{u_{4}}{(0.2,0.3,0.4,0.5)}, \\
& \left.\left.\frac{u_{5}}{(0.0,0.1,0.1,0.2)}\right\},(0.2,0.3,0.4,0.5)\right) \text {, } \\
& \left(\widetilde{F}_{\tilde{f}} \widetilde{\Pi} \widetilde{G}_{\widetilde{g}}\right)\left(e_{3}\right)=\left(\left\{\frac{u_{1}}{(0.1,0.2,0.2,0.3)}, \frac{u_{2}}{(0.5,0.6,0.7,0.8)},\right.\right. \\
& \frac{u_{3}}{(0.4,0.5,0.5,0.6)}, \frac{u_{4}}{(0.2,0.3,0.4,0.5)}, \\
& \left.\left.\frac{u_{5}}{(0.1,0.2,0.2,0.3)}\right\},(0.2,0.3,0.4,0.5)\right) \text {. }
\end{aligned}
$$

Definition 18. A GTFSS is said to be a generalized empty trapezoidal fuzzy soft set, denoted by $\widetilde{\varnothing}$, if $\widetilde{F}_{\tilde{f}}: E \rightarrow T F(U) \times$ $\widetilde{I}$, such that $\widetilde{F}_{\tilde{f}}(e)=(\widetilde{F}(e), \widetilde{f}(e))$, where $\widetilde{F}(e)=\{u /(0,0,0,0)$ : $u \in U\}$ and $\widetilde{f}(e)=(0,0,0,0)$, for all $e \in E$.

Definition 19. A GTFSS is said to be a generalized universal trapezoidal fuzzy soft set, denoted by $\widetilde{U}$, if $\widetilde{F}_{\tilde{f}}: E \rightarrow T F(U) \times$ $\widetilde{I}$, such that $\widetilde{F}_{\tilde{f}}(e)=(\widetilde{F}(e), \widetilde{f}(e))$, where $\widetilde{F}(e)=\{u /(1,1,1,1)$ : $u \in U\}$ and $\tilde{f}(e)=(1,1,1,1)$, for all $e \in E$.

From Definitions 18 and 19, obviously we have
(1) $\widetilde{\varnothing} \sqsubseteq \widetilde{F}_{\widetilde{f}} \sqsubseteq \widetilde{U}$,
(2) $\widetilde{\varnothing}^{c}=\widetilde{U}$,
(3) $\widetilde{U}^{c}=\widetilde{\varnothing}$.

Theorem 20. Let $\widetilde{F}_{\tilde{f}}$ be a GTFSS over $(U, E)$; then the following holds:

(1) $\widetilde{F}_{\tilde{f}} \widetilde{\square} \widetilde{\varnothing}=\widetilde{F}_{\tilde{f}}, \widetilde{F}_{\tilde{f}} \widetilde{\Pi} \widetilde{\varnothing}=\widetilde{\varnothing}$,

(2) $\widetilde{F}_{\tilde{f}} \widetilde{\sqcup} \widetilde{U}=\widetilde{U}, \widetilde{F}_{\tilde{f}} \widetilde{\Pi} \widetilde{U}=\widetilde{F}_{\tilde{f}}$.

Proof. Straightforward.

Remark 21. Let $\widetilde{F}_{\tilde{f}}$ be a GTFSS over $(U, E)$; if $\widetilde{F}_{\tilde{f}} \neq \widetilde{U}$ or $\widetilde{F}_{\tilde{f}} \neq \widetilde{\varnothing}$, then $\widetilde{F}_{\tilde{f}} \widetilde{\sqcup} \widetilde{F}_{\tilde{f}}^{c} \neq \widetilde{U}$ and $\widetilde{F}_{\tilde{f}} \widetilde{\Pi} \widetilde{F}_{\tilde{f}}^{c} \neq \widetilde{\varnothing}$. 
Theorem 22. Let $\widetilde{F}_{\tilde{f}}, \widetilde{G}_{\tilde{g}}$, and $\widetilde{H}_{\tilde{h}}$ be any three GTFSSs over $(U, E)$; then the following holds:

(1) $\widetilde{F}_{\tilde{f}} \widetilde{\sqcup} \widetilde{G}_{\tilde{g}}=\widetilde{G}_{\tilde{g}} \widetilde{\sqcup} \widetilde{F}_{\tilde{f}}$,

(2) $\widetilde{F}_{\tilde{f}} \widetilde{\Pi} \widetilde{G}_{\tilde{g}}=\widetilde{G}_{\tilde{g}} \widetilde{\Pi} \widetilde{F}_{\tilde{f}}$,

(3) $\widetilde{F}_{\tilde{f}} \widetilde{\sqcup}\left(\widetilde{G}_{\tilde{g}} \bar{\square} \widetilde{H}_{\tilde{h}}\right)=\left(\widetilde{F}_{\tilde{f}} \widetilde{\sqcup} \widetilde{G}_{\tilde{g}}\right) \widetilde{\sqcup} \widetilde{H}_{\tilde{h}}$,

(4) $\widetilde{F}_{\tilde{f}} \bar{\Pi}\left(\widetilde{G}_{\tilde{g}} \bar{\Pi} \widetilde{H}_{\tilde{h}}\right)=\left(\widetilde{F}_{\tilde{f}} \widetilde{\Pi} \widetilde{G}_{\tilde{g}}\right) \widetilde{\Pi} \widetilde{H}_{\tilde{h}}$.

Proof. The properties follow from the properties of trapezoidal fuzzy sets.

Theorem 23. Let $\widetilde{F}_{\tilde{f}}$ and $\widetilde{G}_{\tilde{g}}$ be two GTFSSs over $(U, E)$. Then De-Morgan's laws are valid:

(1) $\left(\widetilde{F}_{\tilde{f}} \widetilde{\square} \widetilde{G}_{\tilde{g}}\right)^{c}=\widetilde{F}_{\widetilde{f}}^{c} \widetilde{\Pi} \widetilde{G}_{\widetilde{g}}^{c}$,

(2) $\left(\widetilde{F}_{\tilde{f}} \widetilde{\Pi} \widetilde{G}_{\tilde{g}}\right)^{c}=\widetilde{F}_{\tilde{f}}^{c} \widetilde{\sqcup} \widetilde{G}_{\tilde{g}}^{c}$.

Proof. For all $e \in E$,

$$
\begin{aligned}
\left(\widetilde{F}_{\tilde{f}} \widetilde{\sqcup} \widetilde{G}_{\tilde{g}}\right)^{c} & =(\widetilde{F}(e) \sqcup \widetilde{G}(e), \widetilde{f}(e) \cup \widetilde{g}(e))^{c} \\
& =\left(\widetilde{F}^{c}(e) \sqcap \widetilde{G}^{c}(e), \widetilde{f}^{c}(e) \cap \widetilde{g}^{c}(e)\right) \\
& =\left(\widetilde{F}^{c}(e), \widetilde{f}^{c}(e)\right) \widetilde{\Pi}\left(\widetilde{G}^{c}(e) \sqcap \widetilde{g}^{c}(e)\right)=\widetilde{F}_{\widetilde{f}}^{c} \widetilde{\Pi} \widetilde{G}_{\tilde{g}}^{c} .
\end{aligned}
$$

Likewise, the proof of (2) can be made similarly.

Theorem 24. Let $\widetilde{F}_{\tilde{f}}, \widetilde{G}_{\tilde{g}}$, and $\widetilde{H}_{\tilde{h}}$ be any three GTFSSs over $(U, E)$. Then,

(1) $\widetilde{F}_{\tilde{f}} \widetilde{\square}\left(\widetilde{G}_{\tilde{g}} \widetilde{\Pi} \widetilde{H}_{\tilde{h}}\right)=\left(\widetilde{F}_{\tilde{f}} \widetilde{\square} \widetilde{G}_{\tilde{g}}\right) \widetilde{\Gamma}\left(\widetilde{F}_{\tilde{f}} \widetilde{\sqcup} \widetilde{H}_{\tilde{h}}\right)$,

(2) $\widetilde{F}_{\tilde{f}} \widetilde{\Pi}\left(\widetilde{G}_{\tilde{g}} \widetilde{\square} \widetilde{H}_{\tilde{h}}\right)=\left(\widetilde{F}_{\tilde{f}} \widetilde{\Pi} \widetilde{G}_{\tilde{g}}\right) \widetilde{\square}\left(\widetilde{F}_{\tilde{f}} \widetilde{\Pi} \widetilde{H}_{\tilde{h}}\right)$.

Proof. The proof follows from definition and distributive property of trapezoidal fuzzy sets.

Definition 25. Let $\left(\widetilde{F}_{\tilde{f}}, A\right)$ and $\left(\widetilde{G}_{\tilde{g}}, B\right)$ be two GTFSSs over $(U, E)$. The " $\left(\widetilde{F}_{\tilde{f}}, A\right)$ AND $\left(\widetilde{G}_{\widetilde{g}}, B\right)$," denoted by $\left(\widetilde{F}_{\tilde{f}}, A\right) \wedge$ $\left(\widetilde{G}_{\widetilde{g}}, B\right)$, is defined by $\left(\widetilde{F}_{\tilde{f}}, A\right) \wedge\left(\widetilde{G}_{\widetilde{g}}, B\right)=\left(\widetilde{H}_{\widetilde{h}}, A \times B\right)$, where $\widetilde{H}_{\tilde{h}}(\alpha, \beta)=(\widetilde{H}(\alpha, \beta), \widetilde{h}(\alpha, \beta))$, for all $(\alpha, \beta) \in A \times B$, such that $\widetilde{H}(\alpha, \beta)=\widetilde{F}(\alpha) \sqcap \widetilde{G}(\beta)=\left\{u / \mu_{\widetilde{F}(\alpha)}(u) \cap \mu_{\widetilde{G}(\beta)}(u): u \in U\right\}$ and $\widetilde{h}(\alpha, \beta)=\mu_{\tilde{f}(\alpha)} \cap \mu_{\tilde{g}(\beta)}$.

Definition 26. Let $\left(\widetilde{F}_{\tilde{f}}, A\right)$ and $\left(\widetilde{G}_{\tilde{g}}, B\right)$ be two GTFSSs over $(U, E)$. The “( $\left.\widetilde{F}_{\tilde{f}}, A\right)$ OR $\left(\widetilde{G}_{\tilde{g}}, B\right)$," denoted by $\left(\widetilde{F}_{\tilde{f}}, A\right) \vee\left(\widetilde{G}_{\tilde{g}}, B\right)$, is defined by $\left(\widetilde{F}_{\tilde{f}}, A\right) \vee\left(\widetilde{G}_{\tilde{g}}, B\right)=\left(\widetilde{H}_{\tilde{h}}, A \times B\right)$, where $\widetilde{H}_{\tilde{h}}(\alpha, \beta)=$ $(\widetilde{H}(\alpha, \beta), \widetilde{h}(\alpha, \beta))$, for all $(\alpha, \beta) \in A \times B$, such that $\widetilde{H}(\alpha, \beta)=$ $\widetilde{F}(\alpha) \sqcup \widetilde{G}(\beta)=\left\{u / \mu_{\widetilde{F}(\alpha)}(u) \cup \mu_{\widetilde{G}(\beta)}(u): u \in U\right\}$ and $\widetilde{h}(\alpha, \beta)=$ $\mu_{\tilde{f}(\alpha)} \cup \mu_{\tilde{g}(\beta)}$.
Remark 27. Let $\left(\widetilde{F}_{\tilde{f}}, A\right)$ and $\left(\widetilde{G}_{\tilde{f}}, B\right)$ be two GTFSSs over $(U, E)$. For all $(\alpha, \beta) \in A \times B$, if $\alpha \neq \beta$, then $\left(\widetilde{G}_{\tilde{f}}, B\right) \wedge$ $\left(\widetilde{F}_{\tilde{f}}, A\right) \neq\left(\widetilde{F}_{\tilde{f}}, A\right) \wedge\left(\widetilde{G}_{\tilde{f}}, B\right)$ and $\left(\widetilde{G}_{\tilde{f}}, B\right) \vee\left(\widetilde{F}_{\tilde{f}}, A\right) \neq\left(\widetilde{F}_{\tilde{f}}, A\right) \vee$ $\left(\widetilde{G}_{\tilde{f}}, B\right)$.

Theorem 28. Let $\left(\widetilde{F}_{\tilde{f}}, A\right)$ and $\left(\widetilde{G}_{\tilde{g}}, B\right)$ be two GTFSSs over $(U, E)$. Then

$$
\begin{aligned}
& \text { (1) }\left(\left(\widetilde{F}_{\tilde{f}}, A\right) \wedge\left(\widetilde{G}_{\tilde{g}}, B\right)\right)^{c}=\left(\widetilde{F}_{\tilde{f}}, A\right)^{c} \vee\left(\widetilde{G}_{\tilde{g}}, B\right)^{c}, \\
& \text { (2) }\left(\left(\widetilde{F}_{\tilde{f}}, A\right) \vee\left(\widetilde{G}_{\tilde{g}}, B\right)\right)^{c}=\left(\widetilde{F}_{\tilde{f}}, A\right)^{c} \wedge\left(\widetilde{G}_{\tilde{g}}, B\right)^{c} .
\end{aligned}
$$

Proof. (1) Suppose that $\left(\widetilde{F}_{\tilde{f}}, A\right) \wedge\left(\widetilde{G}_{\tilde{g}}, B\right)=\left(\widetilde{H}_{\tilde{h}}, A \times B\right)$.

Here $\widetilde{H}_{\tilde{h}}^{c}(\alpha, \beta)=\left(\widetilde{H}^{c}(\alpha, \beta), \widetilde{h}^{c}(\alpha, \beta)\right)$, for all $(\alpha, \beta) \in A \times B$. By Definition 25, for all $(\alpha, \beta) \in A \times B$, we have $\widetilde{H}^{c}(\alpha, \beta)=$ $(\widetilde{F}(\alpha) \sqcap \widetilde{G}(\beta))^{c}=\widetilde{F}^{c}(\alpha) \sqcup \widetilde{G}^{c}(\beta)$ and $\widetilde{h}^{c}(\alpha, \beta)=\left(\mu_{\tilde{f}(\alpha)} \cap \mu_{\tilde{g}(\beta)}\right)^{c}=$ $\mu_{\tilde{f}}^{c}(\alpha) \cup \mu_{\tilde{g}}^{c}(\beta)$.

Again suppose that $\left(\widetilde{F}_{\tilde{f}}, A\right)^{c} \vee\left(\widetilde{G}_{\tilde{g}}, B\right)^{c}=\left(\widetilde{O}_{\widetilde{o}}, A \times B\right)$, where $\widetilde{O}_{\tilde{o}}(\alpha, \beta)=(\widetilde{O}(\alpha, \beta), \widetilde{o}(\alpha, \beta))$, for all $(\alpha, \beta) \in A \times B$, such that $\widetilde{O}(\alpha, \beta)=\widetilde{F}^{c}(\alpha) \sqcup \widetilde{G}^{c}(\beta)$ and $\widetilde{o}(\alpha, \beta)=\mu_{\tilde{f}}^{c}(\alpha) \cup \mu_{\widetilde{g}}^{c}(\beta)$. Hence $\widetilde{H}_{\widetilde{h}}^{c}=\widetilde{O}_{\widetilde{o}}$.

Likewise, the proof of (2) can be made similarly.

Theorem 29. Let $\left(\widetilde{F}_{\tilde{f}}, A\right),\left(\widetilde{G}_{\tilde{g}}, B\right)$, and $\left(\widetilde{H}_{\tilde{h}}, C\right)$ be any three GTFSSs over $(U, E)$. Then we have

(1) $\left(\widetilde{F}_{\tilde{f}}, A\right) \wedge\left(\left(\widetilde{G}_{\tilde{g}}, B\right) \wedge\left(\widetilde{H}_{\tilde{h}}, C\right)\right)=\left(\left(\widetilde{F}_{\tilde{f}}, A\right) \wedge\left(\widetilde{G}_{\tilde{g}}, B\right)\right) \wedge$ $\left(\widetilde{H}_{\tilde{h}}, C\right)$,

(2) $\left(\widetilde{F}_{\tilde{f}}, A\right) \vee\left(\left(\widetilde{G}_{\tilde{g}}, B\right) \vee\left(\widetilde{H}_{\tilde{h}}, C\right)\right)=\left(\left(\widetilde{F}_{\tilde{f}}, A\right) \vee\left(\widetilde{G}_{\tilde{g}}, B\right)\right) \vee$ $\left(\widetilde{H}_{\tilde{h}}, C\right)$,

(3) $\left(\widetilde{F}_{\tilde{f}}, A\right) \wedge\left(\left(\widetilde{G}_{\tilde{g}}, B\right) \vee\left(\widetilde{H}_{\tilde{h}}, C\right)\right)=\left(\left(\widetilde{F}_{\tilde{f}}, A\right) \wedge\left(\widetilde{G}_{\tilde{g}}, B\right)\right) \vee$ $\left(\left(\widetilde{F}_{\tilde{f}}, A\right) \wedge\left(\widetilde{H}_{\widetilde{h}}, C\right)\right)$,

(4) $\left(\widetilde{F}_{\tilde{f}}, A\right) \vee\left(\left(\widetilde{G}_{\tilde{g}}, B\right) \wedge\left(\widetilde{H}_{\tilde{h}}, C\right)\right)=\left(\left(\widetilde{F}_{\tilde{f}}, A\right) \vee\left(\widetilde{G}_{\tilde{g}}, B\right)\right) \wedge$ $\left(\left(\widetilde{F}_{\tilde{f}}, A\right) \vee\left(\widetilde{H}_{\tilde{h}}, C\right)\right)$.

Proof. The proof follows from Definitions 25 and 26 and distributive property of trapezoidal fuzzy sets.

In group decision problems, when the attribute of the parameters is imprecise and vague, the generalized trapezoidal fuzzy soft set is more realistic than the trapezoidal fuzzy soft set as each person has various opinions on the vague attributes of the same parameter. In order to further illustrate the point, the following example is given by us.

Example 30. Reconsider Example 8. Each person has different opinions on the vague attributes of the same house. Now Mrs. X also describes the optional five houses under various attributes with linguistic variables intuitively as in Table 3. 
TABLE 3: The ratings of five houses under various attributes for Mrs $\mathrm{X}$.

\begin{tabular}{lccc}
\hline$U$ & Location & Cheap & Size \\
\hline$u_{1}$ & Good & Fair & Medium poor \\
$u_{2}$ & Medium poor & Poor & Medium good \\
$u_{3}$ & Very poor & Medium poor & Fair \\
$u_{4}$ & Medium good & Good & Good \\
$u_{5}$ & Fair & Poor & Very poor \\
$\tilde{g}$ & Good & Fair & Good \\
\hline
\end{tabular}

Similarly to Example 8, we can obtain a corresponding generalized trapezoidal fuzzy soft set $\widetilde{G}_{\tilde{g}}$ as follows:

$$
\begin{gathered}
\widetilde{G}_{\widetilde{g}}\left(e_{1}\right)=\left(\left\{\frac{u_{1}}{(0.7,0.8,0.8,0.9)}, \frac{u_{2}}{(0.2,0.3,0.4,0.5)},\right.\right. \\
\frac{u_{3}}{(0.0,0.1,0.1,0.2)}, \frac{u_{4}}{(0.5,0.6,0.7,0.8)}, \\
\widetilde{G}_{\tilde{g}}\left(e_{2}\right)=\left(\left\{\frac{u_{5}}{(0.4,0.5,0.5,0.6)}\right\},(0.7,0.8,0.8,0.9)\right), \\
\widetilde{G}_{\tilde{g}}\left(e_{3}\right)=\left(\left\{\frac{u_{1}}{(0.4,0.5,0.5,0.6)}, \frac{u_{2}}{(0.2,0.3,0.4,0.5)}, \frac{u_{3}}{(0.7,0.8,0.8,0.9)},\right.\right. \\
\frac{\left.\frac{u_{5}}{(0.1,0.2,0.2,0.3)}\right\},(0.4,0.5,0.5,0.6)}{(0.2,0.3,0.4,0.5)}, \frac{u_{1}}{(0.5,0.6,0.7,0.8)}, \\
\frac{u_{3}}{(0.4,0.5,0.5,0.6)}, \frac{u_{4}}{(0.7,0.8,0.8,0.9)}, \\
\left.\left.\frac{u_{5}}{(0.0,0.1,0.1,0.2)}\right\},(0.7,0.8,0.8,0.9)\right) .
\end{gathered}
$$

Here, we must use AND operation since the different opinions of the married couple have to be considered.

By Definition 25, we can obtain the result of "AND" operation on the generalized trapezoidal fuzzy soft sets $\left(\widetilde{F}_{\tilde{f}}, A\right)$ and $\left(\widetilde{G}_{\tilde{g}}, B\right)$ as follows:

$$
\begin{gathered}
\widetilde{H}_{\tilde{h}}\left(e_{1}, e_{1}\right)=\left(\left\{\frac{u_{1}}{(0.5,0.6,0.7,0.8)}, \frac{u_{2}}{(0.1,0.2,0.2,0.3)},\right.\right. \\
\frac{u_{3}}{(0.0,0.1,0.1,0.2)}, \frac{u_{4}}{(0.4,0.5,0.5,0.6)}, \\
\widetilde{H}_{\tilde{h}}\left(e_{1}, e_{2}\right)=\left(\left\{\frac{u_{5}}{(0.4,0.5,0.5,0.6)}\right\},(0.4,0.5,0.5,0.6)\right), \\
(0.4,0.5,0.5,0.6) \\
\frac{u_{1}}{(0.0,0.1,0.1,0.2)}, \frac{u_{2}}{(0.1,0.2,0.2,0.3)}, \\
\left.\left.\frac{u_{5}}{(0.1,0.2,0.2,0.3)}\right\},(0.4,0.5,0.5,0.5,0.6)\right),
\end{gathered}
$$

$\widetilde{H}_{\widetilde{h}}\left(e_{1}, e_{3}\right)=\left(\left\{\frac{u_{1}}{(0.2,0.3,0.4,0.5)}, \frac{u_{2}}{(0.1,0.2,0.2,0.3)}\right.\right.$,

$$
\frac{u_{3}}{(0.0,0.1,0.1,0.2)}, \frac{u_{4}}{(0.4,0.5,0.5,0.6)},
$$

$$
\left.\left.\frac{u_{5}}{(0.0,0.1,0.1,0.2)}\right\},(0.4,0.5,0.5,0.6)\right) \text {, }
$$

$$
\widetilde{H}_{\widetilde{h}}\left(e_{2}, e_{1}\right)=\left(\left\{\frac{u_{1}}{(0.2,0.3,0.4,0.5)}, \frac{u_{2}}{(0.1,0.2,0.2,0.3)},\right.\right.
$$

$$
\frac{u_{3}}{(0.0,0.1,0.1,0.2)}, \frac{u_{4}}{(0.4,0.5,0.5,0.6)},
$$

$$
\left.\left.\frac{u_{5}}{(0.1,0.2,0.2,0.3)}\right\},(0.5,0.6,0.7,0.8)\right) \text {, }
$$

$$
\widetilde{H}_{\tilde{h}}\left(e_{2}, e_{2}\right)=\left(\left\{\frac{u_{1}}{(0.2,0.3,0.4,0.5)}, \frac{u_{2}}{(0.1,0.2,0.2,0.3)},\right.\right.
$$

$$
\frac{u_{3}}{(0.2,0.3,0.4,0.5)}, \frac{u_{4}}{(0.4,0.5,0.5,0.6)},
$$

$$
\left.\left.\frac{u_{5}}{(0.1,0.2,0.2,0.3)}\right\},(0.4,0.5,0.5,0.6)\right) \text {, }
$$

$$
\begin{aligned}
\widetilde{H}_{\tilde{h}}\left(e_{2}, e_{3}\right)=\left(\left\{\frac{u_{1}}{(0.2,0.3,0.4,0.5)}, \frac{u_{2}}{(0.5,0.6,0.7,0.8)},\right.\right. \\
\frac{u_{3}}{(0.2,0.3,0.4,0.5)}, \frac{u_{4}}{(0.4,0.5,0.5,0.6)}, \\
\left.\left.\frac{u_{5}}{(0.0,0.1,0.1,0.2)}\right\},(0.5,0.6,0.7,0.8)\right),
\end{aligned}
$$

$$
\widetilde{H}_{\widetilde{h}}\left(e_{3}, e_{1}\right)=\left(\left\{\frac{u_{1}}{(0.1,0.2,0.2,0.3)}, \frac{u_{2}}{(0.2,0.3,0.4,0.5)},\right.\right.
$$

$$
\begin{aligned}
& \frac{u_{3}}{(0.0,0.1,0.1,0.2)}, \frac{u_{4}}{(0.5,0.6,0.7,0.8)}, \\
& \left.\left.\frac{u_{5}}{(0.1,0.2,0.2,0.3)}\right\},(0.2,0.3,0.4,0.5)\right),
\end{aligned}
$$

$$
\begin{aligned}
\widetilde{H}_{\widetilde{h}}\left(e_{3}, e_{2}\right)=\left(\left\{\frac{u_{1}}{(0.1,0.2,0.2,0.3)}, \frac{u_{2}}{(0.1,0.2,0.2,0.3)},\right.\right. \\
\frac{u_{3}}{(0.2,0.3,0.4,0.5)}, \frac{u_{4}}{(0.5,0.6,0.7,0.8)}, \\
\left.\left.\frac{u_{5}}{(0.1,0.2,0.2,0.3)}\right\},(0.2,0.3,0.4,0.5)\right),
\end{aligned}
$$

$$
\begin{aligned}
\widetilde{H}_{\tilde{h}}\left(e_{3}, e_{3}\right)=\left(\left\{\frac{u_{1}}{(0.1,0.2,0.2,0.3)}, \frac{u_{2}}{(0.5,0.6,0.7,0.8)},\right.\right. \\
\frac{u_{3}}{(0.4,0.5,0.5,0.6)}, \frac{u_{4}}{(0.5,0.6,0.7,0.8)}, \\
\left.\left.\frac{u_{5}}{(0.0,0.1,0.1,0.2)}\right\},(0.2,0.3,0.4,0.5)\right) .
\end{aligned}
$$


TABLE 4: Defuzzification value of $\widetilde{H}_{\widetilde{h}}\left(e_{i}, e_{j}\right)$.

\begin{tabular}{cccccccccc}
\hline & $\left(e_{1}, e_{1}\right)$ & $\left(e_{1}, e_{2}\right)$ & $\left(e_{1}, e_{3}\right)$ & $\left(e_{2}, e_{1}\right)$ & $\left(e_{2}, e_{2}\right)$ & $\left(e_{2}, e_{3}\right)$ & $\left(e_{3}, e_{1}\right)$ & $\left(e_{3}, e_{2}\right)$ & $\left(e_{3}, e_{3}\right)$ \\
\hline$u_{1}$ & $(0.65)$ & $(0.5)$ & 0.35 & 0.35 & 0.35 & 0.35 & 0.20 & 0.20 & 0.20 \\
$u_{2}$ & 0.20 & 0.20 & 0.20 & 0.43 & 0.2 & $(0.65)$ & 0.35 & 0.2 & $(0.65)$ \\
$u_{3}$ & 0.1 & 0.1 & 0.1 & 0.1 & 0.35 & 0.35 & 0.1 & 0.35 & 0.5 \\
$u_{4}$ & 0.5 & $(0.5)$ & $(0.5)$ & $(0.5)$ & $(0.5)$ & 0.5 & $(0.65)$ & $(0.65)$ & $(0.65)$ \\
$u_{5}$ & 0.5 & 0.2 & 0.1 & 0.2 & 0.2 & 0.1 & 0.2 & 0.2 & 0.1 \\
$\lambda$ & 0.5 & 0.5 & 0.5 & 0.65 & 0.5 & 0.65 & 0.35 & 0.35 & 0.35 \\
\hline
\end{tabular}

TABLE 5: Grade table.

\begin{tabular}{lccccccccc}
\hline & $\left(e_{1}, e_{1}\right)$ & $\left(e_{1}, e_{2}\right)$ & $\left(e_{1}, e_{3}\right)$ & $\left(e_{2}, e_{1}\right)$ & $\left(e_{2}, e_{2}\right)$ & $\left(e_{2}, e_{3}\right)$ & $\left(e_{3}, e_{1}\right)$ & $\left(e_{3}, e_{2}\right)$ & $\left(e_{3}, e_{3}\right)$ \\
\hline$u_{i}$ & $u_{1}$ & $u_{1}, u_{4}$ & $u_{4}$ & $u_{4}$ & $u_{4}$ & $u_{2}$ & $u_{4}$ & $u_{4}$ & $u_{2}, u_{4}$ \\
Highest grade & $\times$ & 0.5 & 0.5 & 0.5 & $\times$ & 0.65 & 0.65 & 0.65 & $\times$ \\
Possibility grade & & 0.5 & 0.5 & 0.65 & & 0.65 & 0.35 & 0.35 & \\
\hline
\end{tabular}

Then, defuzzifying each element of $\widetilde{H}_{\widetilde{h}}\left(e_{i}, e_{j}\right),(i, j=$ $1,2,3)$, by (8), we get a fuzzy matrix showed in Table 4 .

Now to determine the best house which will satisfy the needs of the married couple, we mark the highest numerical grade in each column excluding the last row which is the possibility defuzzification grade of such belongingness of a house against each pair of parameters (see Table 5). Now, the score of each such house is calculated by taking the sum of the products of these numerical grades with the corresponding possibility defuzzification grade $\lambda$. The house with the highest score is the desired one. We do not consider the numerical grades of the house against the pairs $\left(e_{i}, e_{i}\right), i=1,2,3$, as both the parameters are the same:

$$
\begin{aligned}
& \text { Score }\left(u_{1}\right)=0.5 \times 0.5=0.25 \\
& \text { Score }\left(u_{2}\right)=0.65 \times 0.65=0.42, \\
& \text { Score }\left(u_{4}\right)=0.5 \times 0.5+0.5 \times 0.5+0.5 \times 0.65+0.65 \times \\
& 0.35+0.65 \times 0.35=1.28
\end{aligned}
$$

The married couple will select the house with the highest score. Hence, they will buy the house $u_{4}$.

Remark 31. In Example 30, we can note that the generalized trapezoidal fuzzy soft set in the application of group decision problems is more realistic and better than the trapezoidal fuzzy soft set as each person has various opinions on the vague attributes of the same house. For example, Mr. X thinks that the better size of a house is "medium poor," but Mrs. X may not think that. She thinks that the better size of a house is "good." Since it may be impossible or unnecessary to obtain more accurate values, for the subjective judgment of the decision maker and the vagueness of parameters information, piecewise linear trapezoidal membership functions are good enough to capture the vagueness of the attribute of the parameters. Therefore, the generalized trapezoidal fuzzy soft set is effective to express these decision making problems when the attribute of the parameters is imprecise and vague. This makes the decision making based on generalized trapezoidal fuzzy soft sets to be more preferable to reflect the reality.

\section{Application of Generalized Trapezoidal Fuzzy Soft Sets in Medical Diagnosis}

In this section, inspired by Çelik's method to diagnose which patient is suffering from what disease in [27], we also present a method of medical diagnosis based on generalized trapezoidal fuzzy soft sets theory.

Since soft set was introduced by Molodtsov in 1999, soft set and its various extensions have been applied in dealing with practical problems. Especially, applications of fuzzy soft set theory in medical diagnosis problems have been studied by many researchers. De et al. [28] have studied Sanchez's $[29,30]$ method of medical diagnosis using an intuitionistic fuzzy set. Saikia et al. [31] have extended the method in [28] using intuitionistic fuzzy soft set theory. In [32], Chetia and Das have studied Sanchez's approach of medical diagnosis through interval-valued fuzzy soft set obtaining an improvement of the same set presented in De et al. [28]. However, the above existing medical diagnosis approaches could inevitably have their limitations. For example, in the existing approaches we cannot present the precise membership degree how painful a patient's head is. But in the generalized trapezoidal fuzzy soft sets we can solve it. Suppose that a patient tells the doctor that his headache is "very good" with his linguistic assessments. So the doctor knows that the degree of his patient's headache is characterized by a trapezoidal fuzzy number $(0.8,0.9,0.9,1.0)$. Hence, as we know that the membership function of a trapezoidal fuzzy number can express vagueness information caused by linguistic assessments through transforming them into numerical variables objectively, it is natural for us to apply generalized trapezoidal fuzzy soft sets to medical diagnosis in order to capture the vagueness of linguistic assessments information in medical diagnosis.

In the following subsections, we will present the steps and the algorithm for the new approach, respectively, in detail.

4.1. Algorithm of Medical Diagnosis Problem Based on GTFSS. Assume that there is a set of $m$ patients $P=\left\{p_{1}, p_{2}, \ldots, p_{m}\right\}$ 
TABLE 6: Linguistic assessments of three patients.

\begin{tabular}{ccccc}
\hline$U$ & $\begin{array}{c}s_{1}: \\
\text { temperature }\end{array}$ & $s_{2}:$ headache & $s_{3}:$ cough & $\begin{array}{c}s_{4}: \text { stomach } \\
\text { problem }\end{array}$ \\
\hline$p_{1}$ & Fair & $\begin{array}{c}\text { Medium } \\
\text { good }\end{array}$ & $\begin{array}{c}\text { Medium } \\
\text { good }\end{array}$ & Very good \\
$p_{2}$ & Fair & Good & $\begin{array}{c}\text { Medium } \\
\text { good }\end{array}$ & Poor \\
$p_{3}$ & Good & Poor & Fair & Very good \\
\hline
\end{tabular}

with a set of $n$ symptoms $S=\left\{s_{1}, s_{2}, \ldots, s_{n}\right\}$ related to a set of $k$ diseases $D=\left\{d_{1}, d_{2}, \ldots, d_{k}\right\}$.

Thus, we first construct a trapezoidal fuzzy soft set $(\widetilde{F}, P)$ over $S$ where $\widetilde{F}$ is a mapping $\widetilde{F}: P \rightarrow T F(S)$. This trapezoidal fuzzy soft set gives a relation matrix $Q$ called patient-symptom matrix, where the entries are trapezoidal fuzzy numbers $\tilde{a}_{i j}=\left(a_{i j}^{1}, a_{i j}^{2}, a_{i j}^{3}, a_{i j}^{4}\right)$, for $1 \leq i \leq m$ and $1 \leq j \leq n$. The patient-symptom matrix is given as follows:

$$
Q=\begin{gathered}
s_{1} \\
p_{1} \\
\vdots \\
p_{m}
\end{gathered}\left(\begin{array}{cccc}
\tilde{a}_{11} & \tilde{a}_{12} & \cdots & \tilde{a}_{1 n} \\
\tilde{a}_{21} & \tilde{a}_{22} & \cdots & \tilde{a}_{2 n} \\
\vdots & \vdots & \cdots & \vdots \\
\tilde{a}_{m 1} & \tilde{a}_{m 2} & \cdots & \tilde{a}_{m n}
\end{array}\right) .
$$

Secondly, we construct a generalized trapezoidal fuzzy soft set $\left(\widetilde{G}_{\tilde{g}}, S\right)$ over $D$ where $\widetilde{G}_{\tilde{g}}$ is a mapping $\widetilde{G}_{\tilde{g}}$ : $S \rightarrow T F(D) \times \widetilde{I}$. This generalized trapezoidal fuzzy soft set also gives a relation matrix $R$ called symptom-disease matrix, where each element is also taken as trapezoidal fuzzy numbers $\widetilde{b}_{j l}=\left(b_{j l}^{1}, b_{j l}^{2}, b_{j l}^{3}, b_{j l}^{4}\right)$, for $1 \leq j \leq n$ and $1 \leq l \leq k+1$. The symptom-disease matrix is given as follows:

$$
\begin{aligned}
& \begin{array}{lllll}
d_{1} & d_{2} & \cdots & d_{k} & \tilde{g}
\end{array} \\
& R=\begin{array}{c}
s_{1} \\
s_{2} \\
\vdots \\
s_{n}
\end{array}\left(\begin{array}{ccccc}
\tilde{b}_{11} & \tilde{b}_{12} & \cdots & \tilde{b}_{1 k} & \tilde{b}_{1 k+1} \\
\tilde{b}_{21} & \tilde{b}_{22} & \cdots & \tilde{k}_{2 k} & \tilde{k}_{2 k+1} \\
\vdots & \vdots & \cdots & \vdots & \vdots \\
\tilde{b}_{n 1} & \tilde{b}_{n 2} & \cdots & \tilde{b}_{n k} & \tilde{b}_{n k+1}
\end{array}\right) \text {, }
\end{aligned}
$$

where the $i$ th row vector represents $\widetilde{G}_{\widetilde{g}}\left(s_{i}\right)$, the $i$ th column vector represents $d_{i}$, and the last column represents the values of $\tilde{g}$ which is a trapezoidal fuzzy set on $S$.

Thirdly, performing the transformation operation $Q \otimes R$, we get the patient-diagnosis matrix $D$ as follows:

$$
\begin{aligned}
& \begin{array}{lllll}
d_{1} & d_{2} & \cdots & d_{k} & \tilde{g}
\end{array} \\
& D=\begin{array}{c}
p_{1} \\
p_{2} \\
\vdots \\
p_{m}
\end{array}\left(\begin{array}{ccccc}
\widetilde{c}_{11} & \widetilde{c}_{12} & \cdots & \widetilde{c}_{1 k} & \widetilde{c}_{1 k+1} \\
\widetilde{c}_{21} & \widetilde{c}_{22} & \cdots & \widetilde{c}_{2 k} & \widetilde{c}_{2 k+1} \\
\vdots & \vdots & \cdots & \vdots & \vdots \\
\tilde{c}_{m 1} & \widetilde{c}_{m 2} & \cdots & \widetilde{c}_{m k} & \widetilde{c}_{m k+1}
\end{array}\right),
\end{aligned}
$$

where $\widetilde{c}_{i l}=\left(\sum_{j=1}^{n} a_{i j}^{1} b_{i j}^{1}, \sum_{j=1}^{n} a_{i j}^{2} b_{i j}^{2}, \sum_{j=1}^{n} a_{i j}^{3} b_{i j}^{3}, \sum_{j=1}^{n} a_{i j}^{4} b_{i j}^{4}\right),(i=$ $1,2, \ldots, m, l=1,2, \ldots, k+1)$.

Fourthly, defuzzifying each element of the above matrix by (8), we get the fuzzy diagnosis matrix as

$$
D^{*}=\begin{gathered}
d_{1} \\
p_{1} \\
p_{2} \\
\vdots \\
p_{m}
\end{gathered}\left(\begin{array}{ccccc}
v_{11} & v_{12} & \cdots & v_{1 k} & v_{1 k+1} \\
v_{21} & v_{22} & \cdots & v_{2 k} & v_{2 k+1} \\
\vdots & \vdots & \cdots & \vdots & \vdots \\
v_{m 1} & v_{m 2} & \cdots & v_{m k} & v_{m k+1}
\end{array}\right)
$$

Finally, $v_{i l} \geq v_{i, k+1}$ for $1 \leq i \leq m$ and $1 \leq l \leq k$; then we conclude that the patient $p_{i}$ is suffering from disease $d_{l}$.

Therefore, the algorithm of medical diagnosis problem based on GTFSS is as follows.

(1) Input the trapezoidal fuzzy soft set $(\widetilde{F}, P)$ to obtain the patient-symptom matrix $Q$.

(2) Input the generalized trapezoidal fuzzy soft set $\left(\widetilde{G}_{\tilde{g}}, S\right)$ to obtain the symptom-disease matrix $R$.

(3) Perform the transformation operation $Q \otimes R$ to get the patient-diagnosis matrix $D$.

(4) Defuzzify all the elements of the matrix $D$ by (8) to obtain the matrix $D^{*}$.

(5) Find $l$ for which $v_{i l} \geq v_{i, k+1}$ for $1 \leq i \leq m$ and $1 \leq$ $l \leq k$; then we conclude that the patient $p_{i}$ is suffering from disease $d_{l}$.

4.2. A Numerical Example. Suppose there are three patients, John, Tom, and Albert, with symptoms: temperature, headache, cough, and stomach problem. Let the possible diseases relating to the above symptoms be viral fever, typhoid, and malaria. Let $P$ be a set of three patients under consideration of a doctor to diagnose, which is denoted by $P=\left\{p_{1}=\right.$ John, $p_{2}=$ Tom, $p_{3}=$ Albert $\}$. Let $S=\left\{s_{1}, s_{2}, s_{3}, s_{4}\right\}$ be a set of symptoms, where $s_{1}, s_{2}, s_{3}$, and $s_{4}$ represent symptoms: temperature, headache, cough, and stomach problem, respectively. Let $D=\left\{d_{1}, d_{2}, d_{3}\right\}$ be a set of diseases, where $d_{1}, d_{2}$, and $d_{3}$ represent the diseases: viral fever, typhoid, and malaria, respectively.

Now, after talking to the three patients the doctor can construct Table 6 with their linguistic assessments.

Then, we can have a corresponding trapezoidal fuzzy soft set $(\widetilde{F}, P)$ over the universes $S$ through the rule of conversion between linguistic variables and numerical variables showed in Figure 2. This trapezoidal fuzzy soft set $(\widetilde{F}, P)$ can be represented by the relation matrix $Q$, called patient-symptom matrix, and is given as follows: 


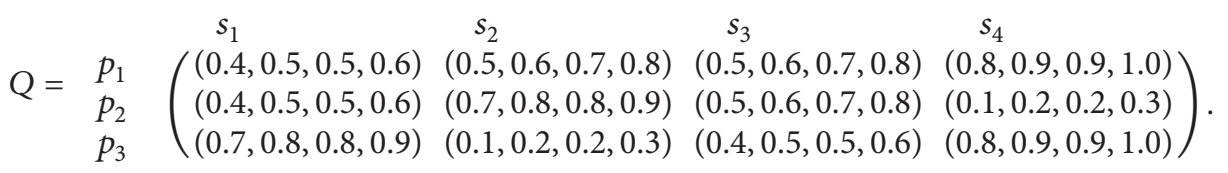

Now we need to construct a generalized trapezoidal fuzzy soft set $\widetilde{F}_{\tilde{f}}$, where $\widetilde{F}_{\tilde{f}}: S \rightarrow T F(D) \times \widetilde{I}$ which is determined from expert medical documentation. Thus the generalized trapezoidal fuzzy soft set $\widetilde{F}_{\tilde{f}}$ gives an approximate description of the three diseases and their symptoms. This generalized trapezoidal fuzzy soft set can be represented by a relation matrix $R$, called symptom-disease matrix, and is given as follows:

$$
\left.\begin{array}{ccccc} 
& d_{1} & d_{2} & d_{3} & \tilde{f} \\
R=s_{1} & (0.8,0.9,0.9,1.0) & (0.5,0.6,0.7,0.8) & (0.7,0.8,0.8,0.9) & (0.5,0.6,0.7,0.8) \\
s_{2} & (0.2,0.3,0.4,0.5) & (0.7,0.8,0.8,0.9) & (0.7,0.8,0.8,0.9) & (0.4,0.5,0.5,0.6) \\
s_{3} & (0.4,0.5,0.5,0.6) & (0.2,0.3,0.4,0.5) & (0.0,0.1,0.1,0.2) & (0.2,0.3,0.4,0.5) \\
s_{4} & (0.0,0.1,0.1,0.2) & (0.7,0.8,0.8,0.9) & (0.5,0.6,0.7,0.8) & (0.7,0.8,0.8,0.9)
\end{array}\right) .
$$

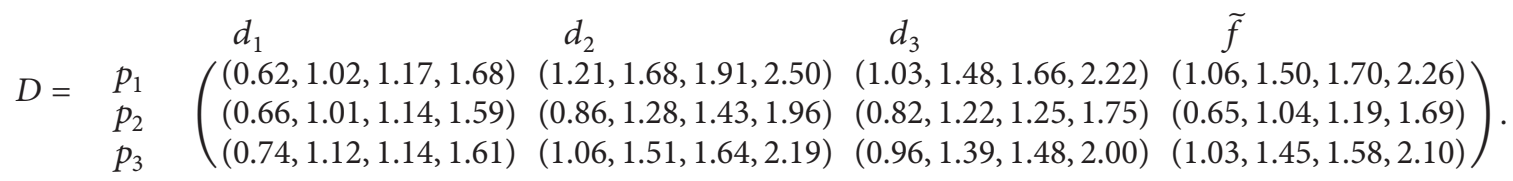

Now, defuzzifying the above matrix, we get

$$
D^{*}=\begin{array}{ccccc}
d_{1} & d_{2} & d_{3} & \tilde{f} \\
p_{1} \\
p_{2} \\
p_{3}
\end{array}\left(\begin{array}{llll}
1.12 & 1.83 & 1.60 & 1.63 \\
1.10 & 1.38 & 1.26 & 1.14 \\
1.15 & 1.60 & 1.46 & 1.54
\end{array}\right) .
$$

From the above matrix we can make the conclusion that John $p_{1}$ and Albert $p_{3}$ are both suffering from the disease typhoid $d_{2}$, but Tom $p_{2}$ is suffering from two diseases typhoid $d_{2}$ and malaria $d_{3}$.

This is only a simple example to show the possibility of using this method for diagnosis of disease which could be improved by incorporating clinical results and other competing diagnoses.

\section{Conclusion}

Soft set theory and trapezoidal fuzzy set are all mathematical tools for dealing with uncertainty. They are closely related. By combining the concept of trapezoidal fuzzy set and soft set models, Xiao et al. [24] presented the concept of the trapezoidal fuzzy soft set which can deal with certain linguistic assessments. Based on [24], we further propose the notion of generalized trapezoidal fuzzy soft set theory. Our generalized trapezoidal fuzzy soft set theory is an extension of trapezoidal fuzzy soft set theory and can help us handle the vagueness of linguistic assessments better. Then some operations are
Then, performing the transformation operation $Q \otimes R$, we get the patient-diagnosis matrix $D$ as follows:

defined on generalized trapezoidal fuzzy soft sets and some of their interesting properties are also discussed. Finally, we give some applications of generalized trapezoidal fuzzy soft set in a decision making problem and medical diagnosis.

In further research, the parameterization reduction of generalized trapezoidal fuzzy soft sets is an important and interesting issue to be addressed.

\section{Conflict of Interests}

The authors declare that there is no conflict of interests regarding the publication of this paper.

\section{Acknowledgments}

The authors would like to thank the anonymous referees for their valuable comments and suggestions. This work is supported by the Natural Science Foundation of China (no. 71261022), the Fundamental Research Funds for the Central Universities of Northwest University for Nationalities (no. 31920130012), and the Young Research Foundation of Northwest University for Nationalities (no. 12XB29).

\section{References}

[1] L. A. Zadeh, "Fuzzy sets," Information and Computation, vol. 8, pp. 338-353, 1965. 
[2] Z. Pawlak, "Rough sets," International Journal of Computer and Information Sciences, vol. 11, no. 5, pp. 341-356, 1982.

[3] D. Molodtsov, "Soft set theory-first results," Computers \& Mathematics with Applications, vol. 37, no. 4-5, pp. 19-31, 1999.

[4] H. Aktaş and N. Çağman, "Soft sets and soft groups," Information Sciences, vol. 177, no. 13, pp. 2726-2735, 2007.

[5] D. Molodtsov, The Theory of Soft Sets, URSS Publishers, Moscow, Russia, 2004 (Russian).

[6] P. K. Maji, R. Biswas, and A. R. Roy, "Soft set theory," Computers \& Mathematics with Applications, vol. 45, no. 4-5, pp. 555-562, 2003.

[7] Y. B. Jun, “Soft BCK/BCI-algebras," Computers \& Mathematics with Applications, vol. 56, no. 5, pp. 1408-1413, 2008.

[8] Y. B. Jun and C. H. Park, "Applications of soft sets in ideal theory of BCK/BCI-algebras," Information Sciences, vol. 178, no. 11, pp. 2466-2475, 2008.

[9] F. Feng, Y. B. Jun, and X. Zhao, "Soft semirings," Computers \& Mathematics with Applications, vol. 56, no. 10, pp. 2621-2628, 2008.

[10] M. I. Ali, F. Feng, X. Liu, W. K. Min, and M. Shabir, "On some new operations in soft set theory," Computers \& Mathematics with Applications, vol. 57, no. 9, pp. 1547-1553, 2009.

[11] K. Qin and Z. Hong, "On soft equality," Journal of Computational and Applied Mathematics, vol. 234, no. 5, pp. 1347-1355, 2010.

[12] J. H. Park, O. H. Kim, and Y. C. Kwun, "Some properties of equivalence soft set relations," Computers \& Mathematics with Applications, vol. 63, no. 6, pp. 1079-1088, 2012.

[13] P. K. Maji, R. Biswas, and A. R. Roy, "Fuzzy soft sets," Journal of Fuzzy Mathematics, vol. 9, no. 3, pp. 589-602, 2001.

[14] P. Majumdar and S. K. Samanta, "Generalised fuzzy soft sets," Computers \& Mathematics with Applications, vol. 59, no. 4, pp. 1425-1432, 2010.

[15] X. Yang, T. Y. Lin, J. Yang, Y. Li, and D. Yu, "Combination of interval-valued fuzzy set and soft set," Computers \& Mathematics with Applications, vol. 58, no. 3, pp. 521-527, 2009.

[16] M. B. Gorzałczany, "A method of inference in approximate reasoning based on interval-valued fuzzy sets," Fuzzy Sets and Systems, vol. 21, no. 1, pp. 1-17, 1987.

[17] G. Deschrijver and E. E. Kerre, "Implicators based on binary aggregation operators in interval-valued fuzzy set theory," Fuzzy Sets and Systems, vol. 153, no. 2, pp. 229-248, 2005.

[18] Y. Yang, X. Tan, and C. Meng, "The multi-fuzzy soft set and its application in decision making," Applied Mathematical Modelling, vol. 37, no. 7, pp. 4915-4923, 2013.

[19] F. Feng, C. Li, B. Davvaz, and M. I. Ali, "Soft sets combined with fuzzy sets and rough sets: a tentative approach," Soft Computing, vol. 14, no. 9, pp. 899-911, 2010.

[20] M. Shabir, M. I. Ali, and T. Shaheen, "Another approach to soft rough sets," Knowledge-Based Systems, vol. 40, pp. 72-80, 2013.

[21] F. Feng, X. Liu, V. Leoreanu-Fotea, and Y. B. Jun, "Soft sets and soft rough sets," Information Sciences, vol. 181, no. 6, pp. 11251137, 2011.

[22] A. Kaur and A. Kumar, "A new method for solving fuzzy transportation problems using ranking function," Applied Mathematical Modelling, vol. 35, no. 12, pp. 5652-5661, 2011.

[23] A. Nieto-Morote and F. Ruz-Vila, "A fuzzy approach to construction project risk assessment," International Journal of Project Management, vol. 29, no. 2, pp. 220-231, 2011.
[24] Z. Xiao, S. Xia, K. Gong, and D. Li, "The trapezoidal fuzzy soft set and its application in MCDM," Applied Mathematical Modelling, vol. 36, no. 12, pp. 5844-5855, 2012.

[25] A. Kaufmann and M. M. Gupta, "Introduction to fuzzy arithmetic: theory and applications: Van Nostrand Reinhold, N. York," International Journal of Approximate Reasoning, vol. 321, pp. 189-190, 1987.

[26] A. Kaufmann and M. M. Gupta, Introduction to Fuzzy Arithmetic: Theory and Applications, Van Nostrand Reinhold, New York, NY, USA, 1991.

[27] Y. Celik and S. Yamak, "Fuzzy soft set theory applied to medical diagnosis using fuzzy arithmetic operations," Journal of Inequalities and Applications, vol. 82, pp. 1-9, 2013.

[28] S. K. De, R. Biswas, and A. R. Roy, "An application of intuitionistic fuzzy sets in medical diagnosis," Fuzzy Sets and Systems, vol. 117, no. 2, pp. 209-213, 2001.

[29] E. Sanchez, "Resolution of composite fuzzy relation equations," Information and Control, vol. 30, no. 1, pp. 38-48, 1976.

[30] E. Sanchez, "Inverses of fuzzy relations. Application to possibility distributions and medical diagnosis," Fuzzy Sets and Systems, vol. 2, no. 1, pp. 75-86, 1979.

[31] B. K. Saikia, P. K. Das, and A. K. Borkakati, "An application of intuitionistic fuzzy soft sets in medical diagnosis," Bio-Science Research Bulletin, vol. 19, no. 2, pp. 121-127, 2003.

[32] B. Chetia and P. K. Das, "An application of interval valued fuzzy soft set in medical diagnosis," International Journal of Contemporary Mathematical Sciences, vol. 5, no. 38, pp. 1887$1894,2010$. 


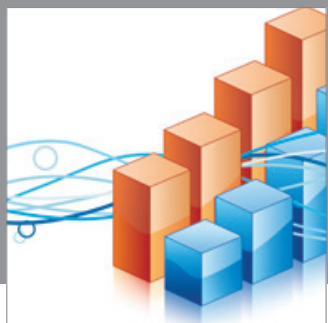

Advances in

Operations Research

mansans

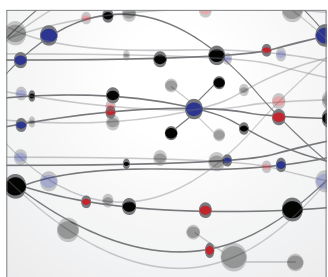

The Scientific World Journal
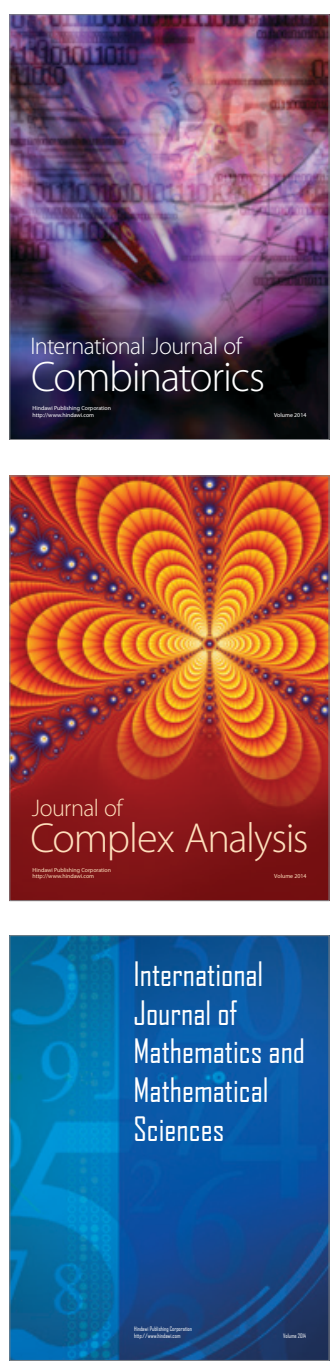
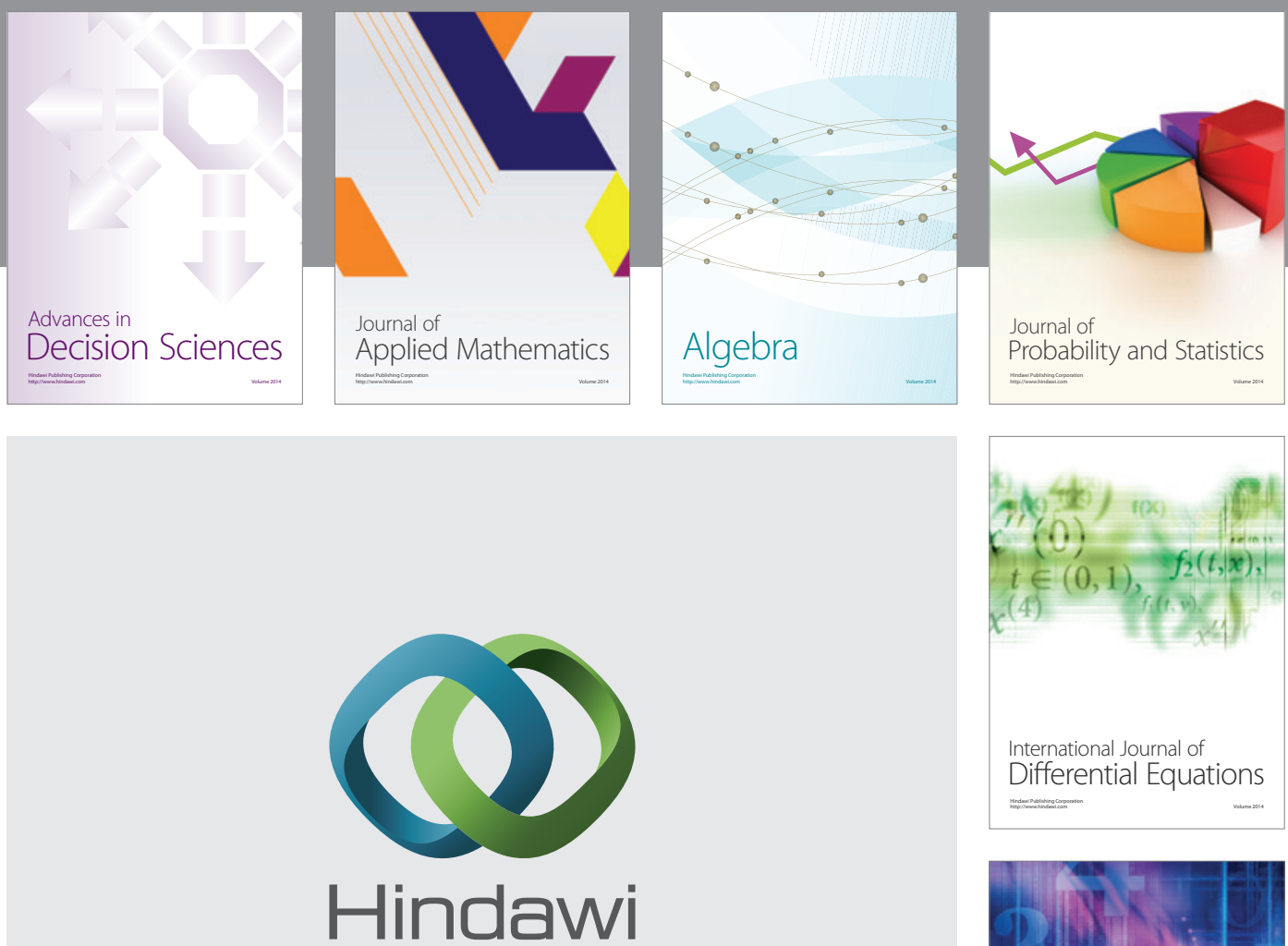

Submit your manuscripts at http://www.hindawi.com
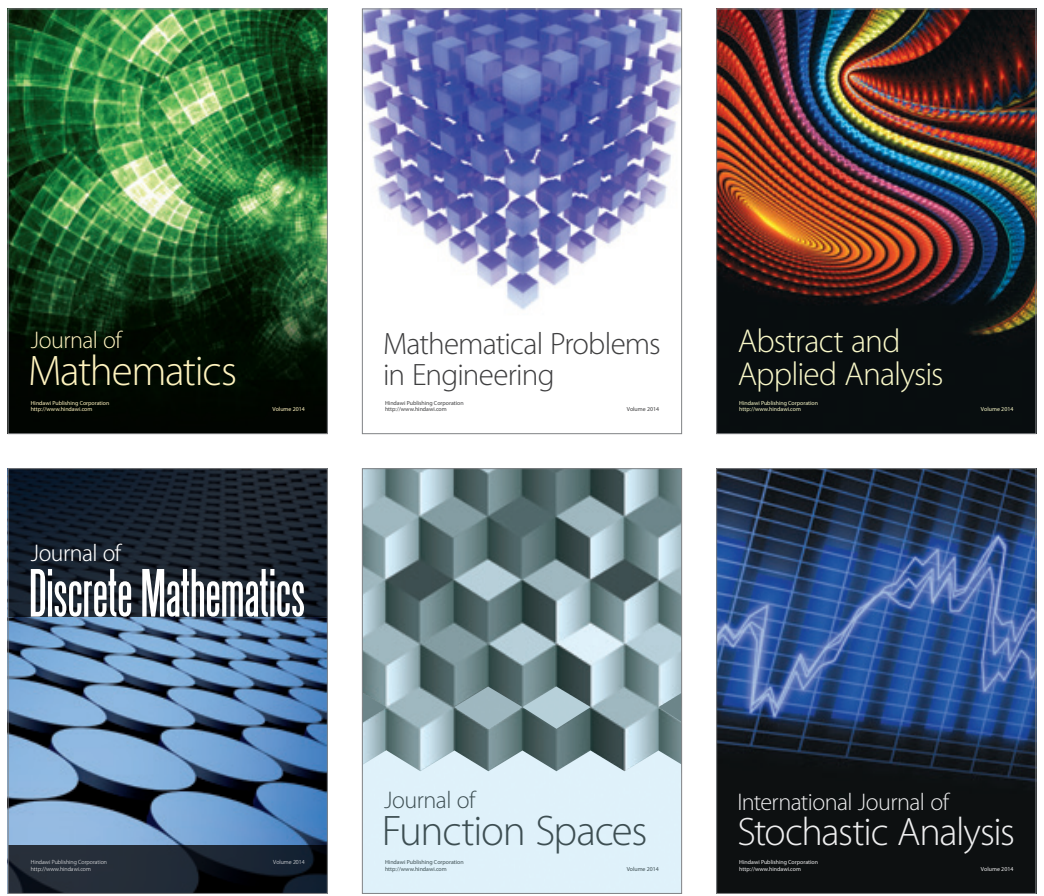

Journal of

Function Spaces

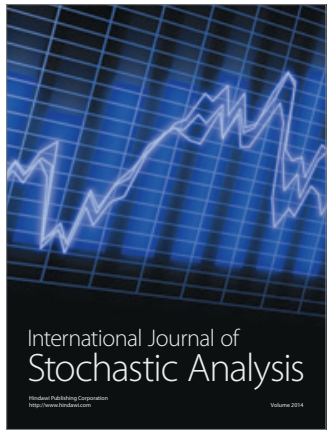

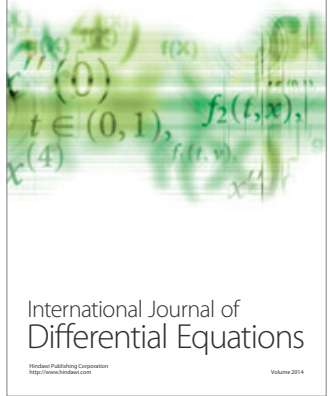
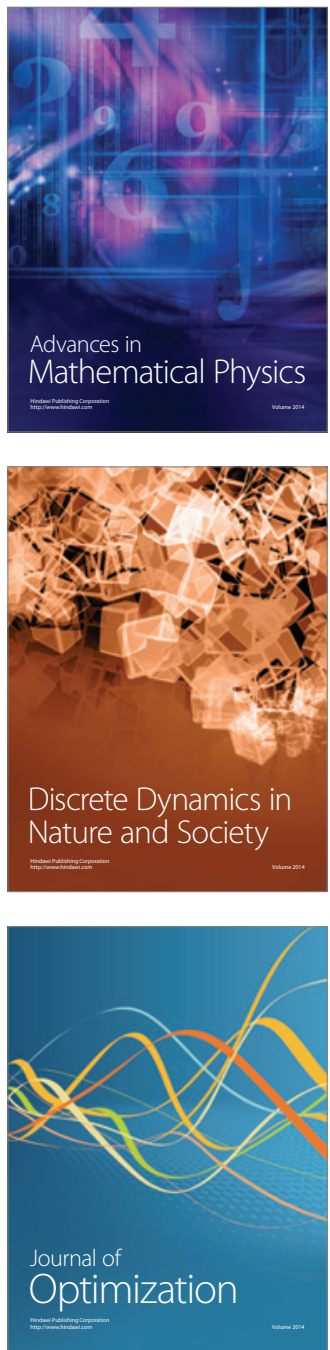